\title{
A Route to 2-Substituted Tetrahydroquinolines via Palladium-Catalyzed Intramolecular Hydroamination of Anilino-alkynes
}

Nitin T. Patil, Huanyou Wu, and Yoshinori Yamamoto*

Department of Chemistry, Graduate School of Science, Tohoku University, Sendai 980-8578, Japan

\section{Contents of Supporting Information}

(28 Pages)

Page S-1: Contents of Supporting Information

Page S-2: Schemes for the Preparation of Substrates 1a-j

Page S-3: Characterization data of Substrates 1a-j

Page S-10: ${ }^{1} \mathrm{H}$ NMR and ${ }^{13} \mathrm{C}$ NMR spectrum of compound 1a

Page S-11: ${ }^{1} \mathrm{H}$ NMR and ${ }^{13} \mathrm{C}$ NMR spectrum of compound $\mathbf{1 b}$

Page S-12: ${ }^{1} \mathrm{H}$ NMR and ${ }^{13} \mathrm{C}$ NMR spectrum of compound 1c

Page S-13: ${ }^{1} \mathrm{H}$ NMR and ${ }^{13} \mathrm{C}$ NMR spectrum of compound $\mathbf{1 d}$

Page S-14: ${ }^{1} \mathrm{H}$ NMR and ${ }^{13} \mathrm{C}$ NMR spectrum of compound $\mathbf{~} \mathbf{g}$

Page S-15: ${ }^{1} \mathrm{H}$ NMR and ${ }^{13} \mathrm{C}$ NMR spectrum of compound $\mathbf{1 h}$

Page S-16: ${ }^{1} \mathrm{H}$ NMR and ${ }^{13} \mathrm{C}$ NMR spectrum of compound $\mathbf{1 i}$

Page S-17: ${ }^{1} \mathrm{H}$ NMR and ${ }^{13} \mathrm{C}$ NMR spectrum of compound $\mathbf{1} \mathbf{j}$

Page S-18: ${ }^{1} \mathrm{H}$ NMR and ${ }^{13} \mathrm{C}$ NMR spectrum of compound 2a

Page S-19: ${ }^{1} \mathrm{H}$ NMR and ${ }^{13} \mathrm{C}$ NMR spectrum of compound $\mathbf{2 b}$

Page S-20: ${ }^{1} \mathrm{H}$ NMR and ${ }^{13} \mathrm{C}$ NMR spectrum of compound $\mathbf{2 c}$

Page S-21: ${ }^{1} \mathrm{H}$ NMR and ${ }^{13} \mathrm{C}$ NMR spectrum of compound $2 d$

Page S-22: ${ }^{1} \mathrm{H}$ NMR and ${ }^{13} \mathrm{C}$ NMR spectrum of compound $\mathbf{2 g}$

Page S-23: ${ }^{1} \mathrm{H}$ NMR and ${ }^{13} \mathrm{C}$ NMR spectrum of compound $\mathbf{2 h}$

Page S-24: ${ }^{1} \mathrm{H}$ NMR and ${ }^{13} \mathrm{C}$ NMR spectrum of compound $\mathbf{2 i}$

Page S-25: ${ }^{1} \mathrm{H}$ NMR and ${ }^{13} \mathrm{C}$ NMR spectrum of compound $\mathbf{2 j}$

Page S-26: ${ }^{1} \mathrm{H}$ NMR and ${ }^{13} \mathrm{C}$ NMR spectrum of compound 3

Page S-27: ${ }^{1} \mathrm{H}$ NMR and ${ }^{13} \mathrm{C}$ NMR spectrum of compound 6

Page S-28: ${ }^{1} \mathrm{H}$ NMR and ${ }^{13} \mathrm{C}$ NMR spectrum of compound 7 
Preparation of the Common Intermediates A, B, and C for the Synthesis of Substrates

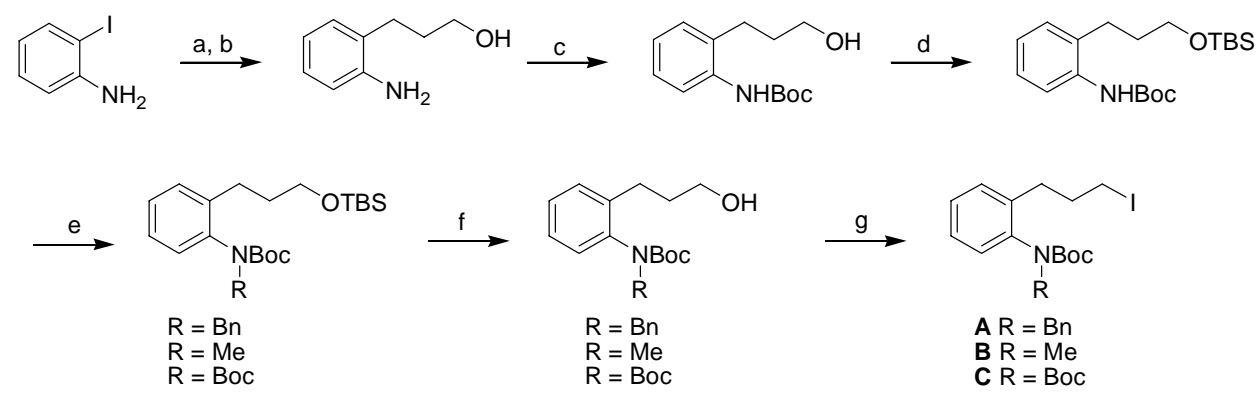

Reagents and Conditions: (a) $2.5 \mathrm{~mol} \% \mathrm{PdCl}_{2}, 3.6 \mathrm{~mol} \% \mathrm{Cul}, 5 \mathrm{~mol} \% \mathrm{PPh}_{3}, 3$ equiv. $\mathrm{Et}_{3} \mathrm{~N}, 1.5$ equiv. propargyl alcohol, $\mathrm{CH}_{3} \mathrm{CN}$, rt, 4h, 90\%; (b) $\mathrm{H}_{2}, 10 \% \mathrm{Pd} / \mathrm{C}, \mathrm{MeOH}$, rt, 48h, 85\%; (c) 1.1 equiv. (Boc) $2 \mathrm{O}$, 2 equiv. $\mathrm{NaHCO}_{3}$, dioxane/ $\mathrm{H}_{2} \mathrm{O}=4: 1$ (v/v), rt, $12 \mathrm{~h}, 85 \%$; (d) 1.1 equiv. TBSCl, 1.1 equiv. imidazole, $\mathrm{CH}_{2} \mathrm{Cl}_{2}$, rt, $2 \mathrm{~h}, 95 \%$; (e) 1.2 equiv. $\mathrm{BnBr} / \mathrm{Mel}, 2 \mathrm{equiv}$. $\mathrm{NaH}$, THF, reflux, $12 \mathrm{~h}, \sim 90 \%$ or 1.1 equiv. (Boc) ${ }_{2} \mathrm{O}, 1.2$ equiv. DMAP, $\mathrm{CH}_{3} \mathrm{CN}$, rt, $12 \mathrm{~h}, 93 \%$; (f) TBAF, THF, $1 \mathrm{~h}, 98 \%$; (g) 2 equiv. $\mathrm{I}_{2}, 2.5$ equiv. $\mathrm{PPh}_{3}, 2.5$ equiv. imidazole, benzene, $\mathrm{rt}, 1 \mathrm{~h}, 80-90 \%$.

Preparation of Substrates 1a-b, 1d, and 1j.

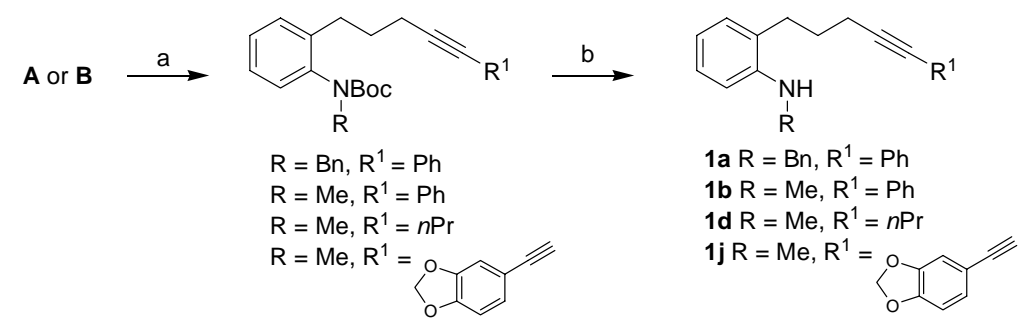

Reagents and Conditions: (a) 1.5 equiv. aryl/alkyl acetylene, 1.5 equiv. $n \mathrm{BuLi}, \mathrm{HMPA}$, THF, $-78^{\circ} \mathrm{C}, 4 \mathrm{~h}, 70-85 \%$; (b) 1.5 equiv. $\mathrm{AcCl}, \mathrm{MeOH}, 0{ }^{\circ} \mathrm{C}, 1 \mathrm{~h}, 90-95 \%$

Scheme for the Preparation of Substrates 1c, 1d, and 1f-i.

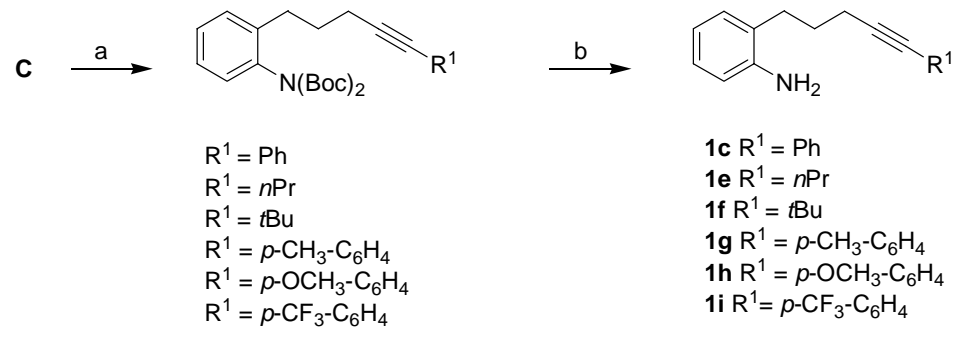

Reagents and Conditions: (a) 1.5 equiv. acetylene, 1.5 equiv. $n \mathrm{BuLi}, \mathrm{HMPA}, \mathrm{THF},-78^{\circ} \mathrm{C}$, 4h, $80-90 \%$; (b) 1.5 equiv. $\mathrm{AcCl}, \mathrm{MeOH}, 0{ }^{\circ} \mathrm{C}, 1 \mathrm{~h}, 90-95 \%$ 


\section{Compound 1a.}

Thick oil; ${ }^{1} \mathrm{H}$ NMR $\left(400 \mathrm{MHz}, \mathrm{CDCl}_{3}\right) \delta$ 7.37-7.25 (m, 10H), $7.09(\mathrm{t}, J=7.6 \mathrm{~Hz}, 2 \mathrm{H}), 6.70(\mathrm{t}, J=7.2$ $\mathrm{Hz}, 1 \mathrm{H}), 6.62(\mathrm{~d}, J=8.0 \mathrm{~Hz}, 1 \mathrm{H}), 4.36(\mathrm{~s}, 2 \mathrm{H}), 4.10(\mathrm{bs}, 1 \mathrm{H}), 2.71(\mathrm{t}, J=7.6 \mathrm{~Hz}, 2 \mathrm{H}), 2.48$ (t, $J=6.8$ $\mathrm{Hz}, 2 \mathrm{H}), 1.97-1.90(\mathrm{~m}, 2 \mathrm{H}) ;{ }^{13} \mathrm{C} \mathrm{NMR}\left(100 \mathrm{MHz}, \mathrm{CDCl}_{3}\right) \delta 145.5,139.4,131.4,129.2,128.5,128.1$, 127.5, 127.1, 125.2, 123.7, 117.2, 110.6, 89.7, 81.3, 48.4, 30.1, 27.8, 19.2; HRMS Calcd for $\mathrm{C}_{24} \mathrm{H}_{23} \mathrm{~N}$ $\left(\mathrm{M}^{+}\right)$325.1830, found $325.1903\left(\mathrm{M}^{+}\right)$. Anal. Calcd for $\mathrm{C}_{24} \mathrm{H}_{23} \mathrm{~N}$ : C, 88.57; H, 7.12; N, 4.30 Found: $\mathrm{C}$, $88.59 ; \mathrm{H}, 7.16 ; \mathrm{N}, 4.35$.

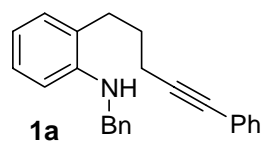

\section{Compound 1b.}

Thick oil; ${ }^{1} \mathrm{H}$ NMR $\left(400 \mathrm{MHz}, \mathrm{CDCl}_{3}\right) \delta 7.4(\mathrm{~m}, 2 \mathrm{H}), 7.27(\mathrm{~m}, 3 \mathrm{H}), 7.15(\mathrm{t}, J=7.6 \mathrm{~Hz}, 1 \mathrm{H}), 7.05(\mathrm{~d}$, $J=7.2 \mathrm{~Hz}, 1 \mathrm{H}), 6.68(\mathrm{t}, J=7.2 \mathrm{~Hz}, 1 \mathrm{H}), 6.60(\mathrm{~d}, J=8.0 \mathrm{~Hz}, 1 \mathrm{H}), 3.80(\mathrm{bs}, 1 \mathrm{H}), 2.83(\mathrm{~s}, 3 \mathrm{H}), 2.65(\mathrm{t}$, $J=7.6 \mathrm{~Hz}, 2 \mathrm{H}), 2.46(\mathrm{t}, J=7.2 \mathrm{~Hz}, 2 \mathrm{H}), 1.89(\mathrm{~m}, 2 \mathrm{H}) ;{ }^{13} \mathrm{C} \mathrm{NMR}\left(100 \mathrm{MHz}, \mathrm{CDCl}_{3}\right) \delta 146.7,131.4$, 129.0, 128.2, 127.6, 127.3, 125.1, 123.7, 116.8, 109.6, 89.9, 81.3, 30.9, 29.9, 27.8, 19.2, HRMS Calcd for $\mathrm{C}_{18} \mathrm{H}_{19} \mathrm{~N}(\mathrm{M}+\mathrm{H})$ 250.1595, found $250.1590(\mathrm{M}+\mathrm{H})$.

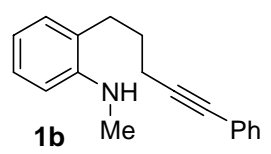

\section{Compound 1c.}

Yellow oil; ${ }^{1} \mathrm{H}$ NMR $\left(400 \mathrm{MHz}, \mathrm{CDCl}_{3}\right) \delta$ 7.38-7.36 (m, 2H), 7.28-7.21 (m, 3H), 7.05-6.98 (m, 2H), $6.70(\mathrm{t}, J=7.2 \mathrm{~Hz}, 1 \mathrm{H}), 6.64(\mathrm{~d}, J=8.0 \mathrm{~Hz}, 1 \mathrm{H}), 3.82(\mathrm{bs}, 2 \mathrm{H}), 2.66$ (t, $J=7.6 \mathrm{~Hz}, 2 \mathrm{H}), 2.43$ (t, $J=$ $6.8 \mathrm{~Hz}, 2 \mathrm{H}), 1.88(\mathrm{~m}, 2 \mathrm{H}) ;{ }^{13} \mathrm{C} \mathrm{NMR}\left(100 \mathrm{MHz}, \mathrm{CDCl}_{3}\right) \delta 144.1,131.4,129.6,128.2,127.6,127.1$, 125.6, 123.7, 118.6, 115.5, 89.8, 81.3, 30.1, 27.9, 19.1; HRMS Calcd for $\mathrm{C}_{17} \mathrm{H}_{17} \mathrm{~N}(\mathrm{M}+\mathrm{H})$ 236.1439, found $236.1434(\mathrm{M}+\mathrm{H})$

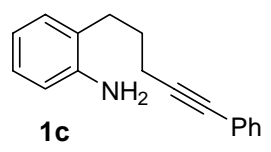

Compound 1d. 
Yellow oil; ${ }^{1} \mathrm{H}$ NMR $\left(400 \mathrm{MHz}, \mathrm{CDCl}_{3}\right) \delta 7.14(\mathrm{t}, J=8.0 \mathrm{~Hz}, 1 \mathrm{H}), 7.03(\mathrm{~d}, J=6.0 \mathrm{~Hz}, 1 \mathrm{H}), 6.67(\mathrm{t}, J$ $=7.2 \mathrm{~Hz}, 1 \mathrm{H}), 6.61(\mathrm{~d}, J=8.0 \mathrm{~Hz}, 1 \mathrm{H}), 3.85(\mathrm{bs}, 1 \mathrm{H}), 2.87$ (s, 3H), 2.59 (t, $J=7.6 \mathrm{~Hz}, 2 \mathrm{H}), 2.23-2.14$ $(\mathrm{m}, 4 \mathrm{H}), 1.77(\mathrm{~m}, 2 \mathrm{H}), 1.58-1.49(\mathrm{~m}, 2 \mathrm{H}), 1.00(\mathrm{t}, J=7.6 \mathrm{~Hz}, 3 \mathrm{H}) ;{ }^{13} \mathrm{C} \mathrm{NMR}\left(100 \mathrm{MHz}, \mathrm{CDCl}_{3}\right) \delta$ 146.8, 129.0, 127.2, 125.4, 116.8, 109.6, 80.9, 79.9, 30.9, 29.9, 28.2, 22.6, 20.9, 18.6, 13.6; HRMS Calcd for $\mathrm{C}_{15} \mathrm{H}_{21} \mathrm{~N}(\mathrm{M}+\mathrm{H})$ 216.1752, found 216. $1747(\mathrm{M}+\mathrm{H})$

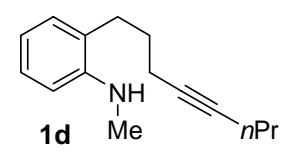

\section{Compound 1g.}

Thick oil; ${ }^{1} \mathrm{H}$ NMR (400 MHz, $\left.\mathrm{CDCl}_{3}\right) \delta 7.29(\mathrm{~d}, J=8.0 \mathrm{~Hz}, 2 \mathrm{H}), 7.10-7.03(\mathrm{~m}, 4 \mathrm{H}), 6.80-6.74(\mathrm{~m}$, 2H), $2.72(\mathrm{t}, J=7.6 \mathrm{~Hz}, 2 \mathrm{H}), 2.46(\mathrm{t}, J=6.8 \mathrm{~Hz}, 2 \mathrm{H}), 2.33(\mathrm{~s}, 3 \mathrm{H}), 1.95-1.88(\mathrm{~m}, 2 \mathrm{H}) ;{ }^{13} \mathrm{C} \mathrm{NMR}(100$ $\left.\mathrm{MHz}, \mathrm{CDCl}_{3}\right) \delta 142.7,137.6,131.3,129.7,128.9,127.1,126.6,120.6,119.7,116.3,89.0,81.4,30.1$, 28.1, 21.4, 19.1; HRMS Calcd for $\mathrm{C}_{18} \mathrm{H}_{19} \mathrm{~N}(\mathrm{M}+\mathrm{H})$ 250.1595, found $250.1590(\mathrm{M}+\mathrm{H})$. Anal. Calcd for $\mathrm{C}_{18} \mathrm{H}_{19} \mathrm{~N}$ : C, 86.70; H, 7.68; N, 5.68 Found: C, 86.65; H, 7.69; N, 5.71.

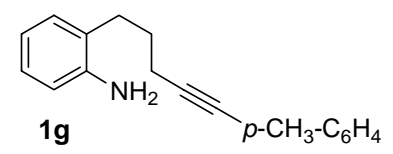

\section{Compound $\mathbf{1 h}$.}

gummy solid; ${ }^{1} \mathrm{H}$ NMR (400 MHz, $\left.\mathrm{CDCl}_{3}\right) \delta 7.34(\mathrm{~d}, J=8.0 \mathrm{~Hz}, 2 \mathrm{H}), 7.08-7.01(\mathrm{~m}, 2 \mathrm{H}), 6.81(\mathrm{~d}, J=$ $7.6 \mathrm{~Hz}, 2 \mathrm{H}), 6.73(\mathrm{t}, J=7.2 \mathrm{~Hz}, 1 \mathrm{H}), 6.66(\mathrm{~d}, J=8.0 \mathrm{~Hz}, 1 \mathrm{H}), 3.79$ (s, 3H), 3.70 (bs, 2H), 2.69 (t, $J=$ $7.2 \mathrm{~Hz}, 2 \mathrm{H}), 2.46(\mathrm{t}, J=6.8 \mathrm{~Hz}, 2 \mathrm{H}), 1.90(\mathrm{~m}, 2 \mathrm{H}) ;{ }^{13} \mathrm{C} \mathrm{NMR}\left(100 \mathrm{MHz}, \mathrm{CDCl}_{3}\right) \delta 159.0,144.1$, 132.8, 129.6, 127.0, 125.7, 118.6, 115.9, 115.5, 113.8, 88.2, 81.0, 55.3, 30.1, 28.0, 19.1; HRMS Calcd for $\mathrm{C}_{18} \mathrm{H}_{19} \mathrm{NO}(\mathrm{M}+\mathrm{H})$ 266.1545, found $266.1539(\mathrm{M}+\mathrm{H})$.

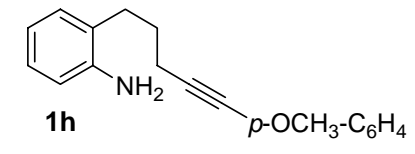

Compound 1i. 
Yellow oil; ${ }^{1} \mathrm{H}$ NMR (400 MHz, $\left.\mathrm{CDCl}_{3}\right) \delta 7.51$ (q, $\left.J=8.4 \mathrm{~Hz}, 4 \mathrm{H}\right), 7.08(\mathrm{q}, 7.2 \mathrm{~Hz}, 2 \mathrm{H}), 6.81(\mathrm{t}, J=$ $7.2 \mathrm{~Hz}, 2 \mathrm{H}), 4.88(\mathrm{bs}, 2 \mathrm{H}), 2.73(\mathrm{t}, J=7.6 \mathrm{~Hz}, 2 \mathrm{H}), 2.50(\mathrm{t}, J=7.2 \mathrm{~Hz}, 2 \mathrm{H}), 1.95(\mathrm{~m}, 2 \mathrm{H}) ;{ }^{13} \mathrm{C}$ NMR $\left(100 \mathrm{MHz}, \mathrm{CDCl}_{3}\right) \delta 144.1,131.7,129.6,128.4,127.6,127.2,125.4,125.1,125.0,118.7,115.6,92.7$, 80.2, 30.1, 27.6, 19.2; HRMS Calcd for $\mathrm{C}_{18} \mathrm{H}_{16} \mathrm{~F}_{3} \mathrm{~N}(\mathrm{M}+\mathrm{H})$ 304.2428, found 304.1308 (M+H). Anal. Calcd for $\mathrm{C}_{18} \mathrm{H}_{16} \mathrm{~F}_{3} \mathrm{~N}$ : C, 71.28; H, 5.32; N, 4.62 Found: C, 71.30; H, 5.36; N, 4.66.

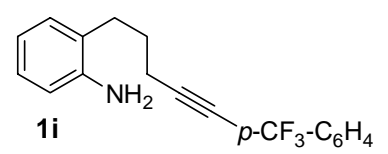

\section{Compound 1j.}

Yellow oil; ${ }^{1} \mathrm{H}$ NMR $\left(400 \mathrm{MHz}, \mathrm{CDCl}_{3}\right) \delta 7.16(\mathrm{t}, J=7.2 \mathrm{~Hz}, 1 \mathrm{H}), 7.06(\mathrm{~d}, J=7.2 \mathrm{~Hz}, 1 \mathrm{H}), 6.93(\mathrm{~d}, J$ $=7.4 \mathrm{~Hz}, 1 \mathrm{H}), 6.86(\mathrm{~s}, 1 \mathrm{H}), 6.73(\mathrm{~d}, J=8.0 \mathrm{~Hz}, 1 \mathrm{H}), 6.69(\mathrm{t}, J=7.2 \mathrm{~Hz}, 1 \mathrm{H}), 6.62(\mathrm{~d}, J=8.0 \mathrm{~Hz}, 1 \mathrm{H})$, $5.95(\mathrm{~s}, 2 \mathrm{H}), 3.83(\mathrm{~s}, 1 \mathrm{H}), 2.86(\mathrm{~s}, 3 \mathrm{H}), 2.65(\mathrm{t}, J=7.2 \mathrm{~Hz}, 2 \mathrm{H}), 2.44(\mathrm{t}, J=6.8 \mathrm{~Hz}, 2 \mathrm{H}), 1.88(\mathrm{~m}, 2 \mathrm{H})$; ${ }^{13} \mathrm{C} \mathrm{NMR}\left(100 \mathrm{MHz}, \mathrm{CDCl}_{3}\right) \delta 147.3,146.7,129.0,127.3,125.8,125.2,117.0,116.8,111.6,109.6$, 108.3, 101.1, 88.0, 81.0, 30.9, 29.9, 27.8, 19.2; HRMS Calcd for $\mathrm{C}_{19} \mathrm{H}_{19} \mathrm{NO}_{2}(\mathrm{M}+\mathrm{H})$ 294.1488, found $294.1489(\mathrm{M}+\mathrm{H})$.

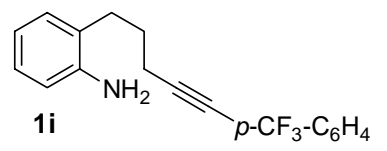

\section{General Procedure for Hydroamination (racemic version).}

To $\mathrm{Pd}\left(\mathrm{PPh}_{3}\right)_{4}(20 \mathrm{~mol} \%)$ and $\mathrm{PhCOOH}(50 \mathrm{~mol} \%)$ was added a substrate $(0.125 \mathrm{mmol})$ in toluene $(1 \mathrm{M})$ under $\mathrm{Ar}$ atmosphere in a screw capped vial. After heating at $120^{\circ} \mathrm{C}$ for the time specified in table 1 , the reaction mixture was filtered through a short silica gel column using diethyl ether as eluent. After evaporation of the solvent, the residue was purified by silica gel column chromatography (hexane-ethyl acetate) to afford the corresponding cyclization product.

\section{General Procedure for Asymmetric Hydroamination.}

To $\mathrm{Pd}_{2}(\mathrm{dba})_{3} \cdot \mathrm{CHCl}_{3}(20 \mathrm{~mol} \%), \operatorname{PhCOOH}(50 \mathrm{~mol} \%)$ and $(R, R)$-RENORPHOS (100 mol \%) was added a substrate $(0.125 \mathrm{mmol})$ in $0.125 \mathrm{ml}$ of toluene under argon atmosphere in a screw capped vial. After heating at $120^{\circ} \mathrm{C}$ for $72 \mathrm{~h}$, the reaction mixture was filtered through a short silica gel column using diethyl ether as eluent. After evaporation of the solvent, the residue was purified by 
silica gel column chromatography (hexane-ethyl acetate) to afford the corresponding cyclization product.

\section{Compound $\mathbf{2 a}$.}

Thick oil; ${ }^{1} \mathrm{H}$ NMR (400 MHz, $\left.\mathrm{CDCl}_{3}\right) \delta$ 7.31-7.19 (m, 10H), 6.98 (m, 2H), 6.59 (t, J= 7.2 Hz, 1H), $6.49(\mathrm{~d}, J=8.0 \mathrm{~Hz}, 1 \mathrm{H}), 6.41(\mathrm{~d}, J=15.6 \mathrm{~Hz}, 1 \mathrm{H}), 6.22(\mathrm{q}, J=7.2 \mathrm{~Hz}, 1 \mathrm{H}), 4.66(\mathrm{~d}, J=17.6 \mathrm{~Hz}, 1 \mathrm{H})$, $4.42(\mathrm{~d}, J=17.6 \mathrm{~Hz}, 1 \mathrm{H}), 4.13(\mathrm{q}, J=6.1 \mathrm{~Hz}, 1 \mathrm{H}), 2.95-2.88(\mathrm{~m}, 1 \mathrm{H}), 2.76-2.72(\mathrm{~m}, 1 \mathrm{H}), 2.19-2.03$ $(\mathrm{m}, 2 \mathrm{H}) ;{ }^{13} \mathrm{C} \mathrm{NMR}\left(100 \mathrm{MHz}, \mathrm{CDCl}_{3}\right) \delta 144.6,138.9,136.7,133.7,133.5,130.9,130.3,128.7,128.6$, 128.5, 128.4, 128.3, 127.4, 127.2, 126.6, 126.3, 121.9, 115.7, 110.9, 59.8, 52.6, 27.7, 24.5; HRMS

Calcd for $\mathrm{C}_{24} \mathrm{H}_{23} \mathrm{~N}(\mathrm{M}+\mathrm{Na}) 348.1728$, found $348.1723(\mathrm{M}+\mathrm{Na}) ;[\alpha]^{20}{ }_{\mathrm{D}}=-21.2\left(c 0.85, \mathrm{CHCl}_{3}\right)$. Anal. Calcd for $\mathrm{C}_{24} \mathrm{H}_{23} \mathrm{~N}$ : C, 88.57; H, 7.12; N, 4.30 Found: C, 88.60; H, 7.17; N, 4.34.

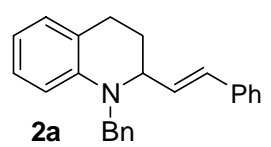

\section{Compound $\mathbf{2 b}$.}

Thick oil; ${ }^{1} \mathrm{H}$ NMR $\left(400 \mathrm{MHz}, \mathrm{CDCl}_{3}\right) \delta$ 7.35-7.18 (m, 5H), $7.11(\mathrm{t}, J=7.6 \mathrm{~Hz}, 1 \mathrm{H}), 6.97(\mathrm{~d}, J=7.2$ $\mathrm{Hz}, 1 \mathrm{H}), 6.62(\mathrm{~m}, 2 \mathrm{H}), 6.43(\mathrm{~d}, J=15.6 \mathrm{~Hz}, 1 \mathrm{H}), 6.18(\mathrm{q}, J=7.2 \mathrm{~Hz}, 1 \mathrm{H}), 3.95(\mathrm{q}, J=6.1 \mathrm{~Hz}, 1 \mathrm{H})$, $2.92(\mathrm{~s}, 3 \mathrm{H}), 2.87-2.67(\mathrm{~m}, 2 \mathrm{H}), 2.10-2.04(\mathrm{~m}, 1 \mathrm{H}), 1.99-1.93(\mathrm{~m}, 1 \mathrm{H}) ;{ }^{13} \mathrm{C} \mathrm{NMR}\left(100 \mathrm{MHz}, \mathrm{CDCl}_{3}\right)$ $\delta 145.4,136.8,130.7,128.5,128.4,127.3,127.2,126.3,122.5,115.7,110.4,61.4,37.4,27.8,24.6$; $(\mathrm{M}+\mathrm{H}) \mathrm{HRMS}$ Calcd for $\mathrm{C}_{18} \mathrm{H}_{19} \mathrm{~N}(\mathrm{M}+\mathrm{H}) 250.1595$, found $250.1590(\mathrm{M}+\mathrm{H})$.

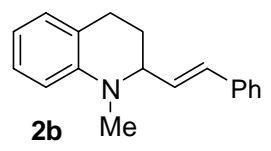

\section{Compound 2c.}

Gummy solid; ${ }^{1} \mathrm{H}$ NMR (400 MHz, $\left.\mathrm{CDCl}_{3}\right) \delta$ 7.38-7.20 (m, 5H), $7.00(\mathrm{~m}, 2 \mathrm{H})$, 6.64-6.56 (m, 2H), $6.51(\mathrm{~d}, J=7.6 \mathrm{~Hz}, 1 \mathrm{H}), 6.24(\mathrm{q}, J=7.2 \mathrm{~Hz}, 1 \mathrm{H}), 4.04-3.99(\mathrm{~m}, 1 \mathrm{H}), 3.90(\mathrm{bs}, 1 \mathrm{H}), 2.89-2.74(\mathrm{~m}, 2 \mathrm{H})$, 2.10-2.03 (m, 1H), 1.89-1.80 (m, 1H); $\left.{ }^{13} \mathrm{C} \mathrm{NMR} \mathrm{(100} \mathrm{MHz,} \mathrm{CDCl}_{3}\right) \delta 143.9,136.7,132.1,130.3$, 
129.2, 128.5, 127.5, 126.8, 126.3, 120.9, 117.1, 114.0, 54.1, 28.6, 25.9; HRMS Calcd for $\mathrm{C}_{17} \mathrm{H}_{17} \mathrm{~N}$ $(\mathrm{M}+\mathrm{H}) 236.1439$, found $236.1434(\mathrm{M}+\mathrm{H}) ;[\alpha]^{20}{ }_{\mathrm{D}}=+25.1\left(c 0.45, \mathrm{CHCl}_{3}\right)$.

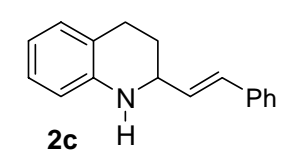

Compound 2d.

Thick oil; ${ }^{1} \mathrm{H}$ NMR $\left(400 \mathrm{MHz}, \mathrm{CDCl}_{3}\right) \delta 7.07$ (t, $\left.J=7.6 \mathrm{~Hz}, 1 \mathrm{H}\right), 6.93(\mathrm{~d}, J=7.2 \mathrm{~Hz}, 1 \mathrm{H}), 6.59-6.54$ $(\mathrm{m}, 2 \mathrm{H}), 5.48(\mathrm{~m}, 1 \mathrm{H}), 5.37(\mathrm{q}, J=6.8 \mathrm{~Hz}, 1 \mathrm{H}), 3.70(\mathrm{q}, J=6.4 \mathrm{~Hz}, 1 \mathrm{H}), 2.84(\mathrm{~s}, 3 \mathrm{H}), 2.79-2.62(\mathrm{~m}$, $2 \mathrm{H}), 2.01-1.93(\mathrm{~m}, 3 \mathrm{H}), 1.86-1.81(\mathrm{~m}, 1 \mathrm{H}), 1.41-1.32(\mathrm{~m}, 2 \mathrm{H}), 0.87(\mathrm{t}, J=7.2 \mathrm{~Hz}, 3 \mathrm{H}) ;{ }^{13} \mathrm{C}$ NMR $\left(100 \mathrm{MHz}, \mathrm{CDCl}_{3}\right) \delta 145.7,131.7,130.6,128.3,127.0,122.5,115.4,110.2,61.1,37.1,34.3,27.9$, 24.5, 22.5, 13.7; HRMS Calcd for $\mathrm{C}_{15} \mathrm{H}_{21} \mathrm{~N}(\mathrm{M}+\mathrm{H}) 216.1752$, found $216.1747(\mathrm{M}+\mathrm{H}) \cdot[\alpha]^{20}{ }_{\mathrm{D}}=+20.1$ (c $0.45, \mathrm{CHCl}_{3}$ ).

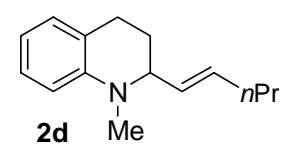

Compound 2g.

Yellow oil; ${ }^{1} \mathrm{H}$ NMR $\left(400 \mathrm{MHz}, \mathrm{CDCl}_{3}\right) \delta 7.26(\mathrm{t}, J=8.0 \mathrm{~Hz}, 2 \mathrm{H}), 7.11(\mathrm{~d}, J=8.0 \mathrm{~Hz}, 2 \mathrm{H}), 6.98(\mathrm{t}, J$ $=8.0 \mathrm{~Hz}, 2 \mathrm{H}), 6.64-6.50(\mathrm{~m}, 3 \mathrm{H}), 6.19(\mathrm{q}, J=7.2 \mathrm{~Hz}, 1 \mathrm{H}), 4.02-3.91(\mathrm{~m}, 1 \mathrm{H}), 3.96(\mathrm{~s}, 1 \mathrm{H}), 2.89-2.73$ (m, 2H), $2.40(\mathrm{~s}, 3 \mathrm{H}), 2.09-2.02(\mathrm{~m}, 1 \mathrm{H}), 1.89-1.79(\mathrm{~m}, 1 \mathrm{H}) ;{ }^{13} \mathrm{C} \mathrm{NMR}\left(100 \mathrm{MHz}, \mathrm{CDCl}_{3}\right) \delta 144.0$, 137.3, 133.9, 131.1, 130.2, 129.2, 129.1, 126.8, 120.9, 117.1, 114.0, 54.1, 28.6, 25.9, 21.2; HRMS Calcd for $\mathrm{C}_{18} \mathrm{H}_{19} \mathrm{~N}(\mathrm{M}+\mathrm{H}) 250.1595$, found $250.1590(\mathrm{M}+\mathrm{H})$.

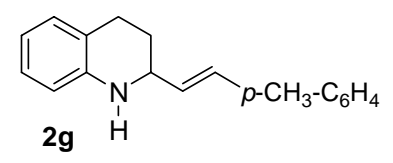

Compound 2h.

Thick oil; ${ }^{1} \mathrm{H}$ NMR $\left(400 \mathrm{MHz}, \mathrm{CDCl}_{3}\right) \delta 7.30(\mathrm{~d}, J=8.8 \mathrm{~Hz}, 2 \mathrm{H}), 6.98(\mathrm{t}, J=8.0 \mathrm{~Hz}, 2 \mathrm{H}), 6.84(\mathrm{~d}, J$ $=8.8 \mathrm{~Hz}, 2 \mathrm{H}), 6.62(\mathrm{t}, J=4.4 \mathrm{~Hz}, 1 \mathrm{H}), 6.51(\mathrm{t}, J=8.4 \mathrm{~Hz}, 2 \mathrm{H}), 6.10(\mathrm{q}, J=7.2 \mathrm{~Hz}, 1 \mathrm{H}), 4.00-3.96(\mathrm{~m}$, $1 \mathrm{H}), 3.79(\mathrm{bs}, 1 \mathrm{H}), 3.80(\mathrm{~s}, 3 \mathrm{H}), 2.89-2.73(\mathrm{~m}, 2 \mathrm{H}), 2.08-2.02(\mathrm{~m}, 1 \mathrm{H}), 1.88-1.79(\mathrm{~m}, 1 \mathrm{H}) ;{ }^{13} \mathrm{C} \mathrm{NMR}$ $\left(100 \mathrm{MHz}, \mathrm{CDCl}_{3}\right) \delta 150.1,144.0,129.9,129.8,129.5,129.1,127.4,126.8,120.9,117.1,114.0$, 113.9, 55.3, 54.2, 28.7, 26.0; HRMS Calcd for $\mathrm{C}_{18} \mathrm{H}_{19} \mathrm{NO}(\mathrm{M}+\mathrm{H}) 266.1545$, found $266.1539(\mathrm{M}+\mathrm{H})$. 


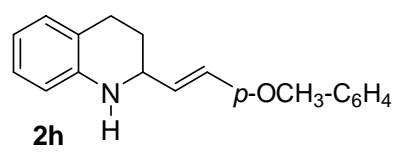

Compound 2i.

Thick oil; ${ }^{1} \mathrm{H}$ NMR (400 MHz, $\left.\mathrm{CDCl}_{3}\right) \delta 7.55(\mathrm{~d}, J=8.1 \mathrm{~Hz}, 2 \mathrm{H}), 7.45(\mathrm{~d}, J=8.3 \mathrm{~Hz}, 2 \mathrm{H}), 7.00(\mathrm{~m}$, 2H), 6.66-6.59 (m, 2H), $6.53(\mathrm{~d}, J=7.8 \mathrm{~Hz}, 1 \mathrm{H}), 6.35(\mathrm{q}, J=7.2 \mathrm{~Hz}, 1 \mathrm{H}), 4.08-4.04(\mathrm{~m}, 1 \mathrm{H}), 3.93$ (bs, $1 \mathrm{H}), 2.89-2.74(\mathrm{~m}, 2 \mathrm{H}), 2.12-2.04(\mathrm{~m}, 1 \mathrm{H}), 1.91-1.82(\mathrm{~m}, 1 \mathrm{H}),{ }^{13} \mathrm{C}$ NMR $\left(100 \mathrm{MHz}, \mathrm{CDCl}_{3}\right) \delta 143.7$, 140.2, 134.9, 129.4, 129.2, 129.1, 128.9, 126.9, 126.4, 125.5, 125.4, 120.8, 117.3, 114.1, 53.8, 28.3, 25.6; HRMS Calcd for $\mathrm{C}_{18} \mathrm{H}_{16} \mathrm{~F}_{3} \mathrm{~N}(\mathrm{M}+\mathrm{H}) 304.1313$, found 304.1308. Anal. Calcd for $\mathrm{C}_{18} \mathrm{H}_{16} \mathrm{~F}_{3} \mathrm{~N}$ : C, 71.28; H, 5.32; N, 4.62 Found: C, 71.29; H, 5.37; N, 4.65.

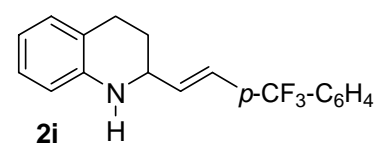

Compound 2j.

Thick oil; ${ }^{1} \mathrm{H}$ NMR $\left(400 \mathrm{MHz}, \mathrm{CDCl}_{3}\right) \delta 7.10(\mathrm{t}, J=6.8 \mathrm{~Hz}, 1 \mathrm{H}), 6.96(\mathrm{~d}, J=6.6 \mathrm{~Hz}, 1 \mathrm{H}), 6.88$, (s, $1 \mathrm{H}), 6.75(\mathrm{q}, J=8.0 \mathrm{~Hz}, 2 \mathrm{H}), 6.61(\mathrm{t}, J=7.2 \mathrm{~Hz}, 2 \mathrm{H}), 6.34(\mathrm{~d}, J=15.6 \mathrm{~Hz}, 1 \mathrm{H}), 6.00(\mathrm{q}, J=7.2 \mathrm{~Hz}$, $1 \mathrm{H}), 5.95(\mathrm{~s}, 2 \mathrm{H}), 3.94-3.90(\mathrm{~m}, 1 \mathrm{H}), 3.90(\mathrm{~s}, 3 \mathrm{H}), 2.88-2.65(\mathrm{~m}, 2 \mathrm{H}), 2.10-1.90(\mathrm{~m}, 2 \mathrm{H}) ;{ }^{13} \mathrm{C} \mathrm{NMR}$ $\left(100 \mathrm{MHz}, \mathrm{CDCl}_{3}\right) \delta 147.9,146.9,145.4,131.3,130.2,128.9,128.4,127.1,122.4,120.8,115.7$, 110.4, 108.1, 105.6, 100.9, 61.3, 37.3, 27.8, 24.5; HRMS Calcd for $\mathrm{C}_{19} \mathrm{H}_{19} \mathrm{NO}_{2}(\mathrm{M}+\mathrm{H})$ 294.1494, found $294.1489(\mathrm{M}+\mathrm{H})$. Anal. Calcd for $\mathrm{C}_{19} \mathrm{H}_{19} \mathrm{NO}_{2}$ : C, 77.79; H, 6.53; N, 4.77 Found: $\mathrm{C}, 77.82 ; \mathrm{H}$, $6.58 ; \mathrm{N}, 4.74$.

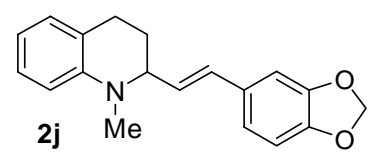

\section{General Procedure for Hydrogenation.}

A mixture of the compound $\mathbf{2 d}$ (or $\mathbf{2 j}$ ) $(0.5 \mathrm{mmol})$ and $10 \% \mathrm{Pd} / \mathrm{C}$ (10\% by weight) in methanol (10 $\mathrm{mL}$ ) was hydrogenated at balloon pressure for $12 \mathrm{~h}$. The catalyst was filtered through Celite and washed with methanol. The solvent was evaporated to give thick oil which was purified by column chromatography (hexane/ethyl acetate) to give the product 3,6 or $\mathbf{7}$. 


\section{Compound 3.}

Thick oil; ${ }^{1} \mathrm{H}$ NMR (400 MHz, $\left.\mathrm{CDCl}_{3}\right) \delta$ 7.30-7.17 (m, 5H), $6.94(\mathrm{t}, J=7.2 \mathrm{~Hz}, 2 \mathrm{H}), 6.58(\mathrm{t}, J=7.2$ $\mathrm{Hz}, 1 \mathrm{H}), 6.42(\mathrm{~d}, J=7.8 \mathrm{~Hz}, 1 \mathrm{H}), 3.73(\mathrm{bs}, 1 \mathrm{H}), 3.28(\mathrm{~m}, 1 \mathrm{H}), 2.84-2.71(\mathrm{~m}, 4 \mathrm{H}), 2.00-1.96(\mathrm{~m}, 1 \mathrm{H})$, $1.82(\mathrm{q}, J=6.8 \mathrm{~Hz}, 2 \mathrm{H}), 1.71-1.62(\mathrm{~m}, 1 \mathrm{H}) ;{ }^{13} \mathrm{C} \mathrm{NMR}\left(100 \mathrm{MHz}, \mathrm{CDCl}_{3}\right) \delta 144.4,141.7,129.1$, 128.4, 128.3, 126.6, 125.9, 121.2, 117.0, 114.1, 51.1, 38.3, 32.2, 28.0, 26.3; HRMS Calcd for $\mathrm{C}_{19} \mathrm{H}_{19} \mathrm{NO}_{2}(\mathrm{M}+\mathrm{H}) 238.1595$, found $238.1590(\mathrm{M}+\mathrm{H}) ;[\alpha]^{26}{ }_{\mathrm{D}}=+52.3\left(c 0.55, \mathrm{CHCl}_{3}\right)$.

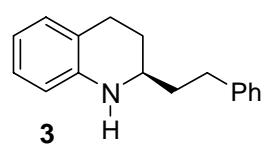

\section{Compound 6.}

Thick oil; ${ }^{1} \mathrm{H}$ NMR $\left(400 \mathrm{MHz}, \mathrm{CDCl}_{3}\right) \delta 6.99(\mathrm{t}, J=7.6 \mathrm{~Hz}, 1 \mathrm{H}), 6.88(\mathrm{~d}, J=7.2 \mathrm{~Hz}, 1 \mathrm{H}), 6.49(\mathrm{t}, J=$ $7.6 \mathrm{~Hz}, 1 \mathrm{H}), 6.43(\mathrm{~d}, J=8.0 \mathrm{~Hz}, 1 \mathrm{H}), 3.14(\mathrm{~m}, 1 \mathrm{H}), 2.84(\mathrm{~s}, 3 \mathrm{H}), 2.76-2.68(\mathrm{~m}, 1 \mathrm{H}), 2.57(\mathrm{t}, J=4.4$ $\mathrm{Hz}, 1 \mathrm{H}), 1.85-1.74(\mathrm{~m}, 2 \mathrm{H}), 1.55-1.46(\mathrm{~m}, 1 \mathrm{H}), 1.36-1.14(\mathrm{~m}, 7 \mathrm{H}), 0.82(\mathrm{t}, J=6.8 \mathrm{~Hz}, 3 \mathrm{H}) ;{ }^{13} \mathrm{C} \mathrm{NMR}$ $\left(100 \mathrm{MHz}, \mathrm{CDCl}_{3}\right) \delta$ 145.3, 128.5, 127.0, 121.8, 115.1, 110.3, 59.0, 38.0, 32.1, 31.2, 25.8, 24.5, 23.6, 22.7, 14.1; HRMS Calcd for $\mathrm{C}_{15} \mathrm{H}_{23} \mathrm{~N}(\mathrm{M}+\mathrm{H}) 218.1908$, found $218.1903(\mathrm{M}+\mathrm{H}) .[\alpha]^{26}{ }_{\mathrm{D}}=-3.5(c 0.50$, $\left.\mathrm{CHCl}_{3}\right)$.

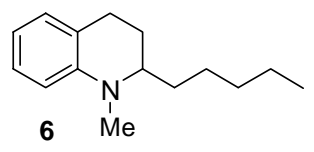

\section{Compound 7.}

Thick oil; ${ }^{1} \mathrm{H}$ NMR (400 MHz, $\left.\mathrm{CDCl}_{3}\right) \delta 7.06(\mathrm{t}, J=7.2 \mathrm{~Hz}, 1 \mathrm{H}), 6.96(\mathrm{~d}, J=7.2 \mathrm{~Hz}, 1 \mathrm{H}), 6.71(\mathrm{~d}, J$ $=7.8 \mathrm{~Hz}, 1 \mathrm{H}), 6.66(\mathrm{~s}, 1 \mathrm{H}), 6.63-6.56(\mathrm{~m}, 2 \mathrm{H}), 6.51(\mathrm{~d}, J=8.0 \mathrm{~Hz}, 1 \mathrm{H}), 5.80(\mathrm{~s}, 2 \mathrm{H}), 3.28-3.23(\mathrm{~m}$, $1 \mathrm{H}), 2.90(\mathrm{~s}, 3 \mathrm{H}), 2.85-2.80(\mathrm{~m}, 1 \mathrm{H}), 2.53-2.45(\mathrm{~m}, 3 \mathrm{H}), 1.96-1.83(\mathrm{~m}, 3 \mathrm{H}), 1.74-1.64(\mathrm{~m}, 1 \mathrm{H}) ;{ }^{13} \mathrm{C}$ NMR (100 MHz, $\left.\mathrm{CDCl}_{3}\right) \delta 147.5,145.5,145.2,135.7,128.6,127.0,121.7,120.9,115.4,110.6,108.6$, 108.1, 100.7, 58.2, 38.1, 33.2, 32.1, 24.5, 23.6; HRMS Calcd for $\mathrm{C}_{19} \mathrm{H}_{21} \mathrm{NO}_{2}(\mathrm{M}+\mathrm{H})$ 296.1650, found $296.1645(\mathrm{M}+\mathrm{H})$.

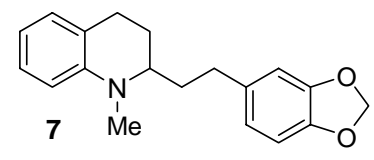




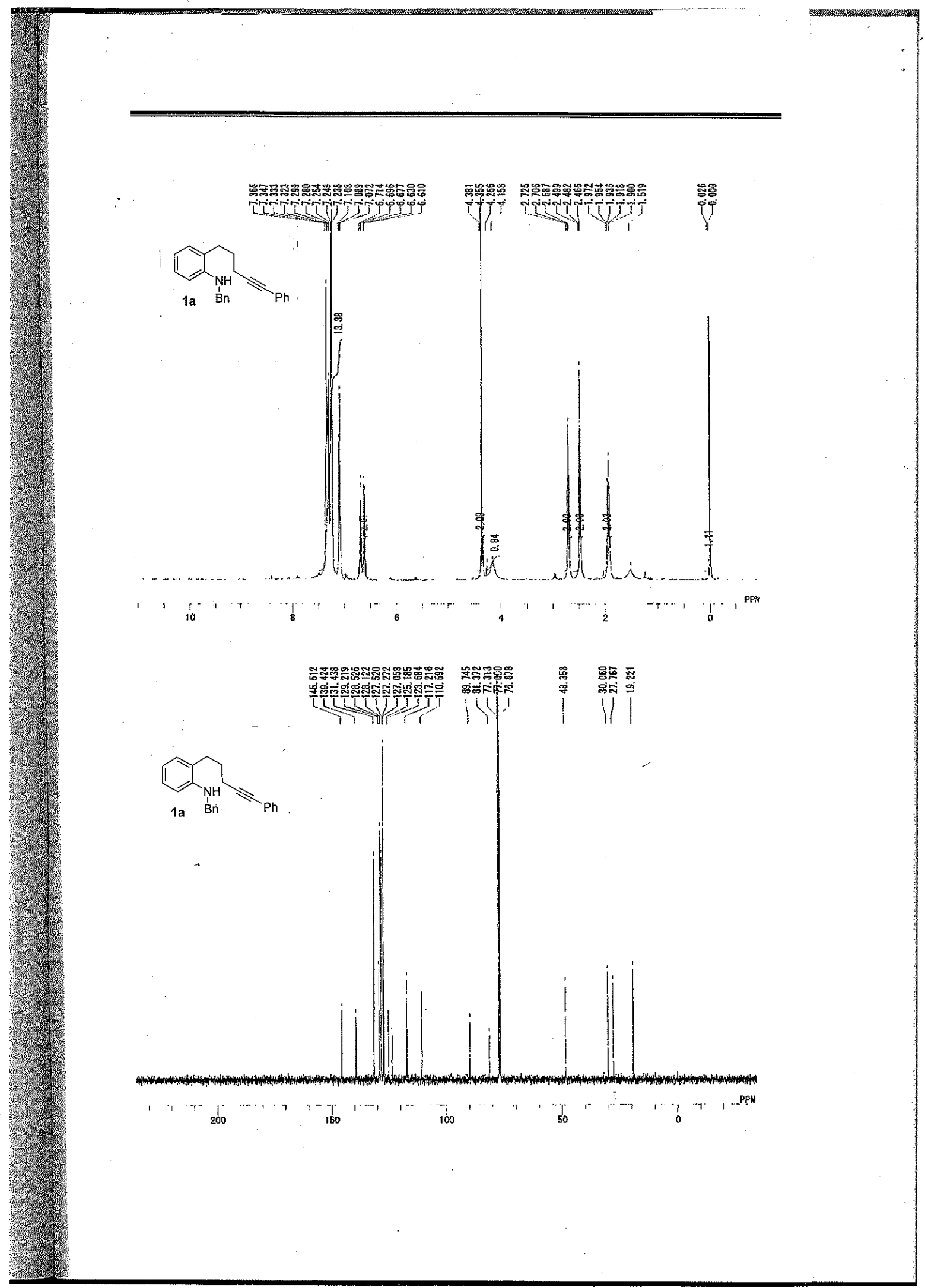




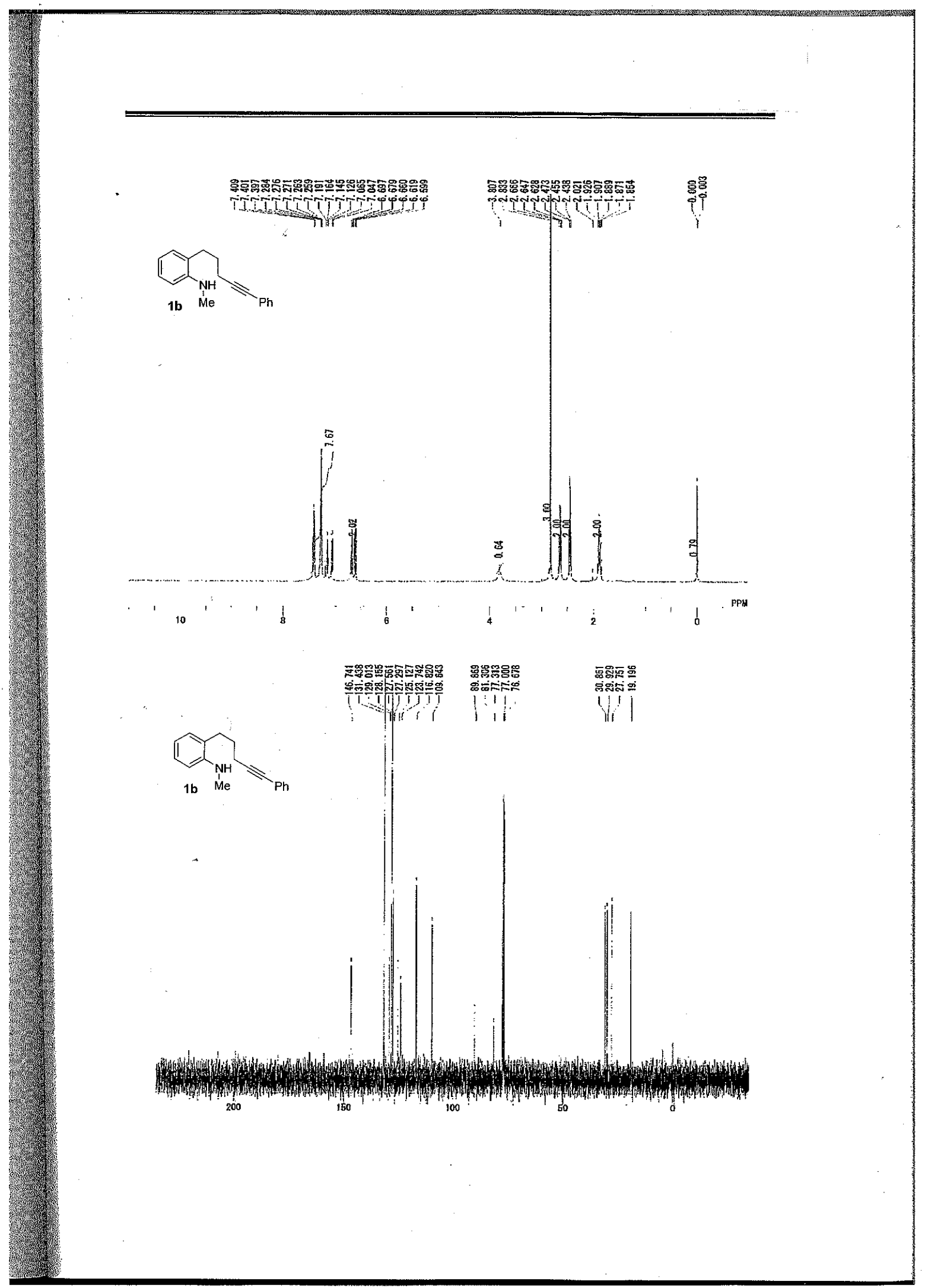




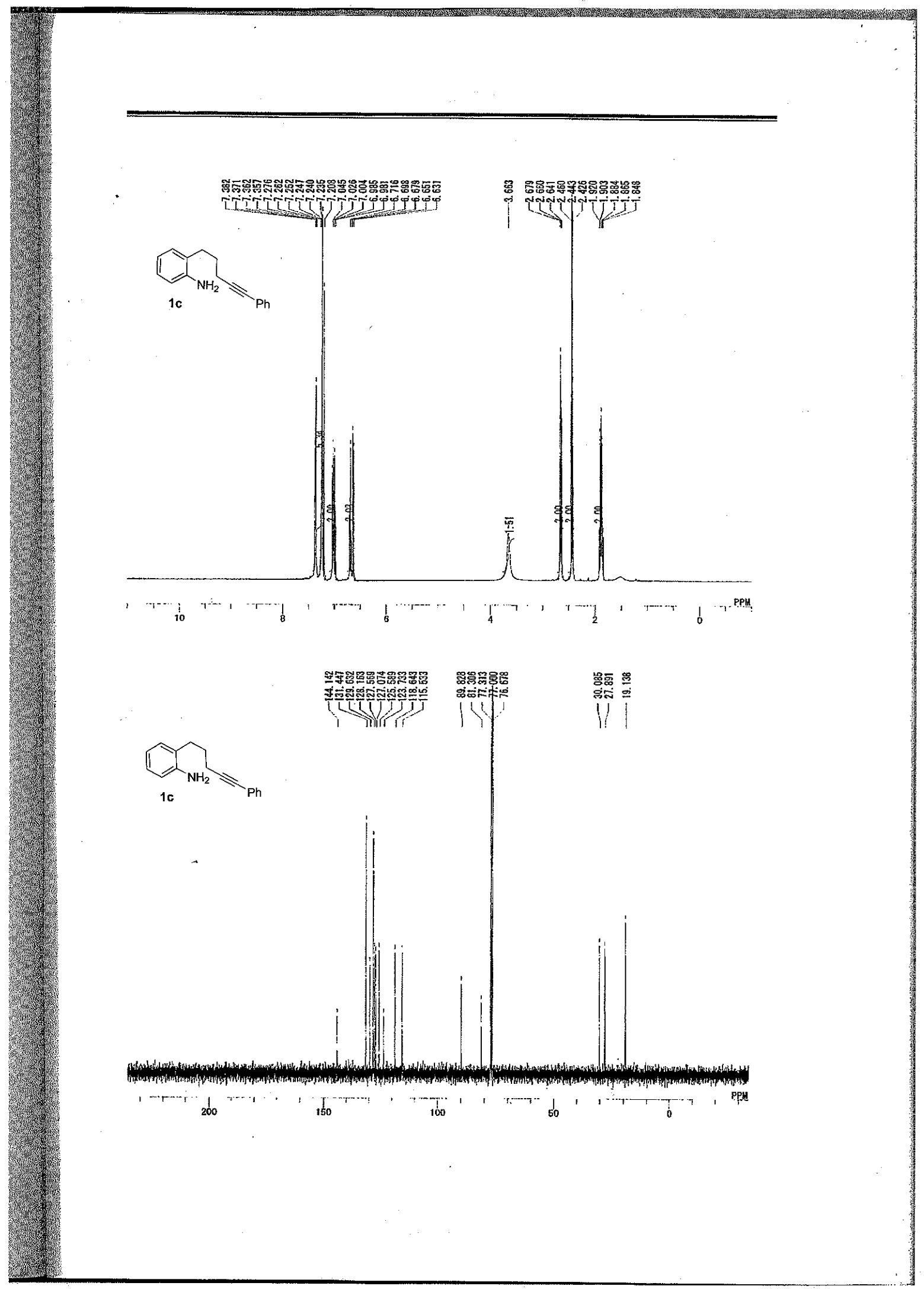




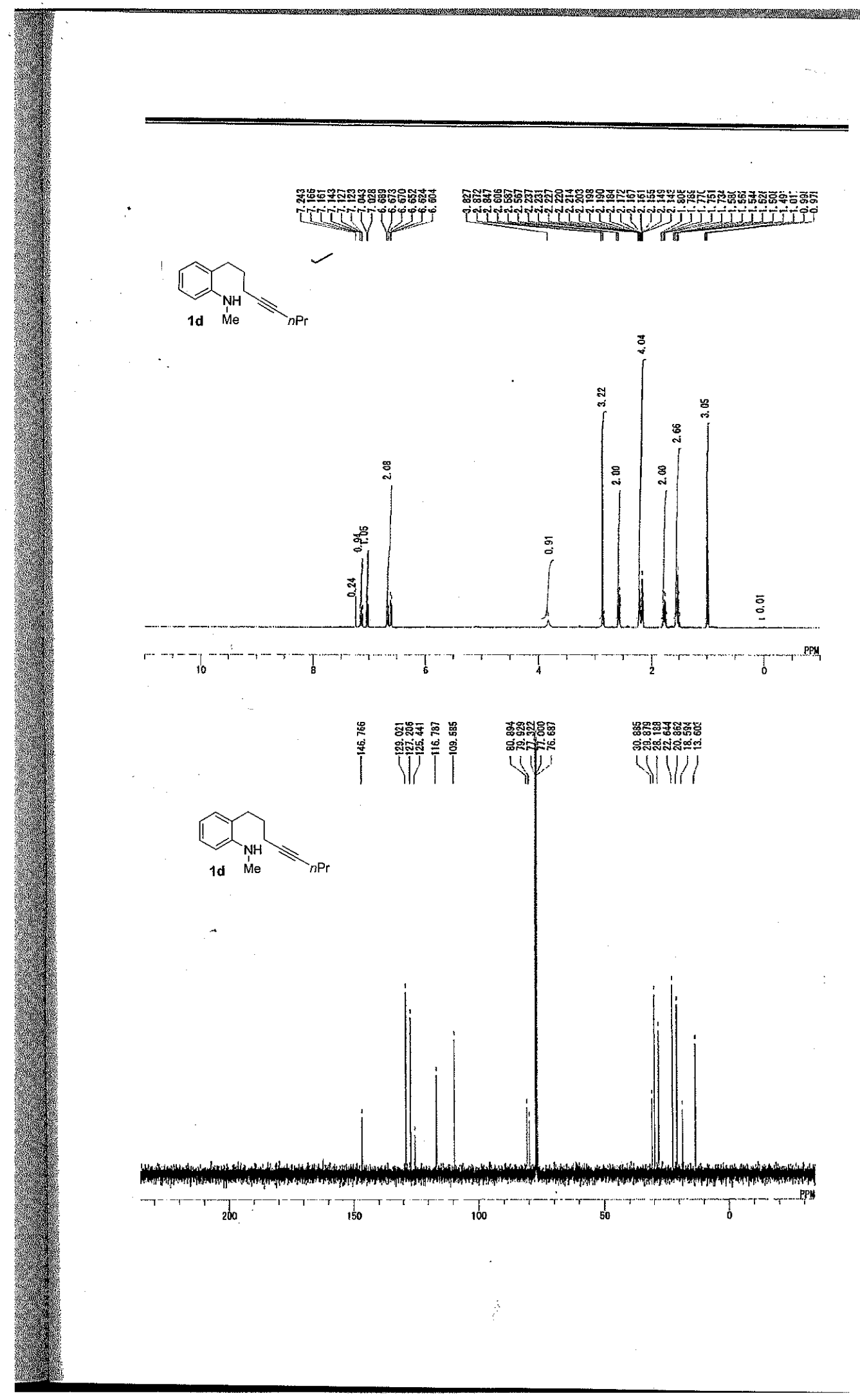




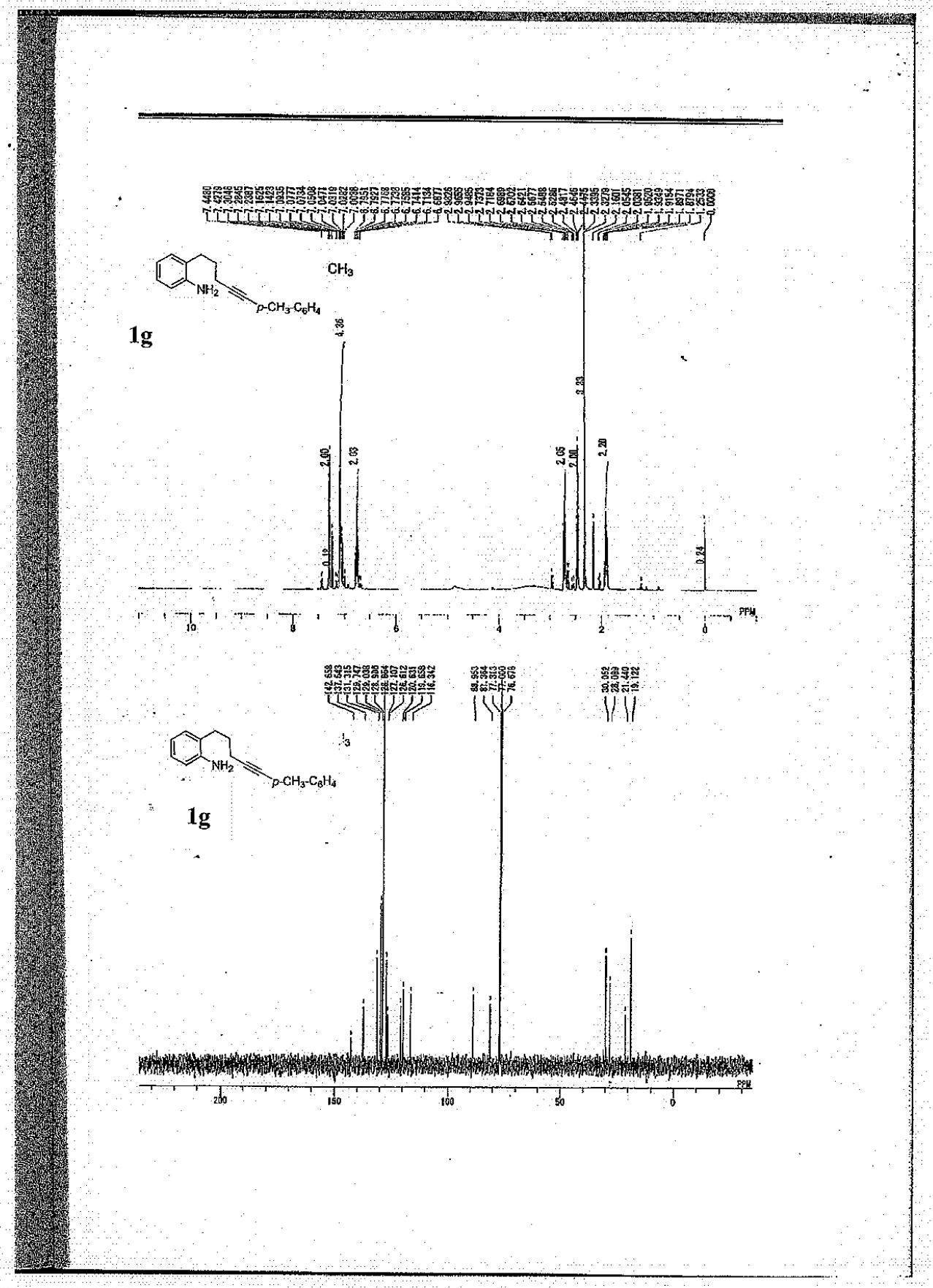




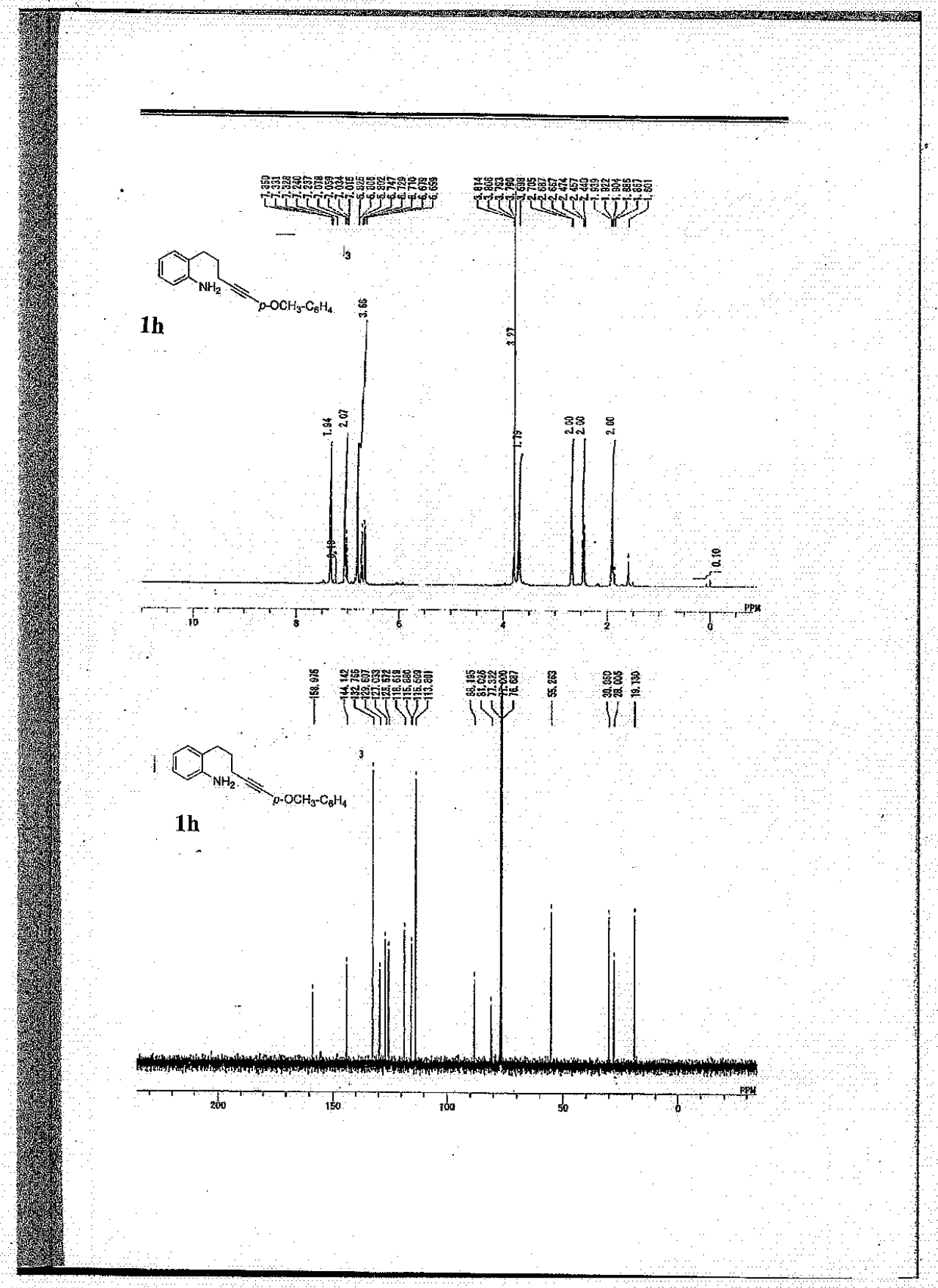




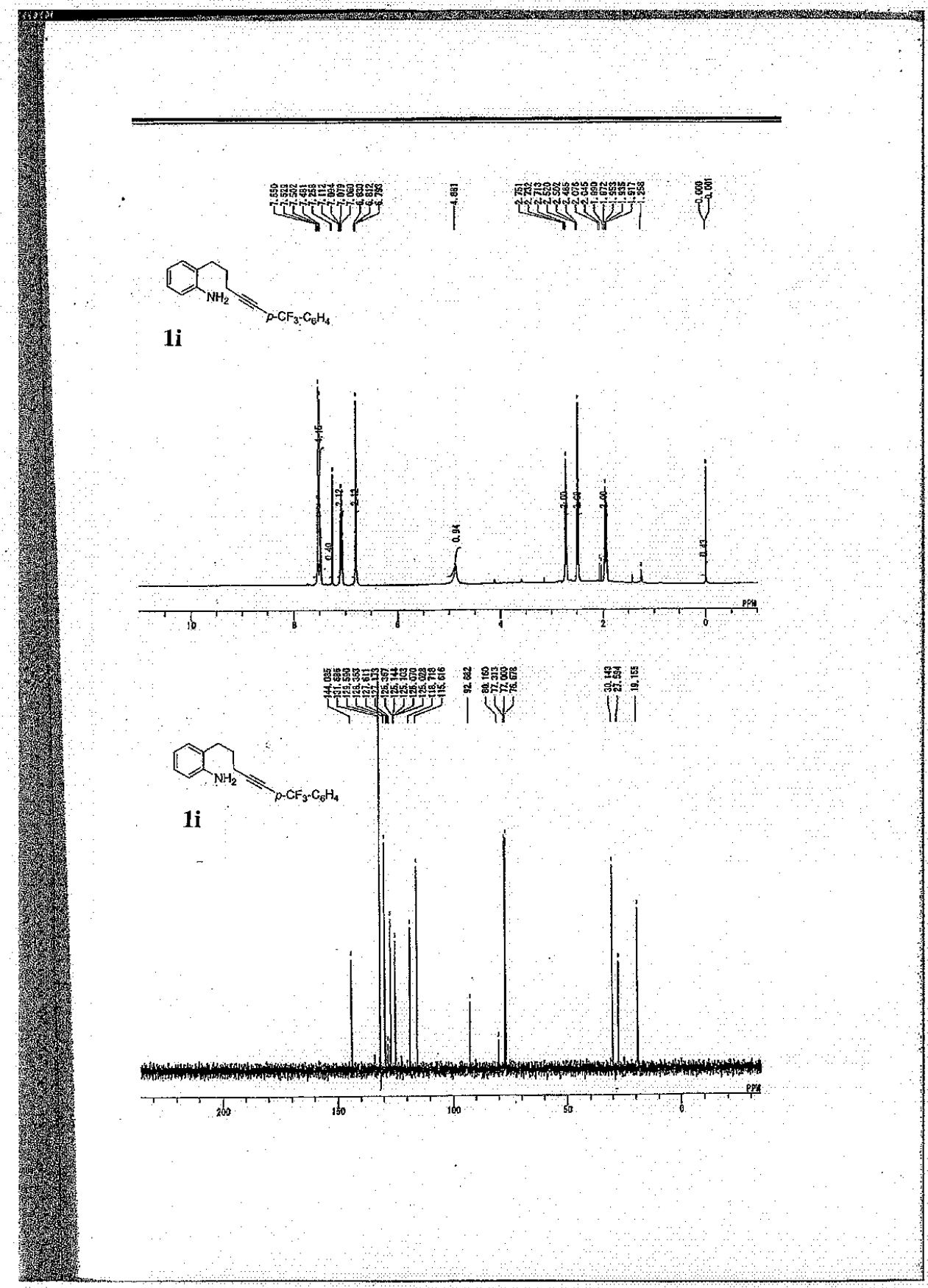




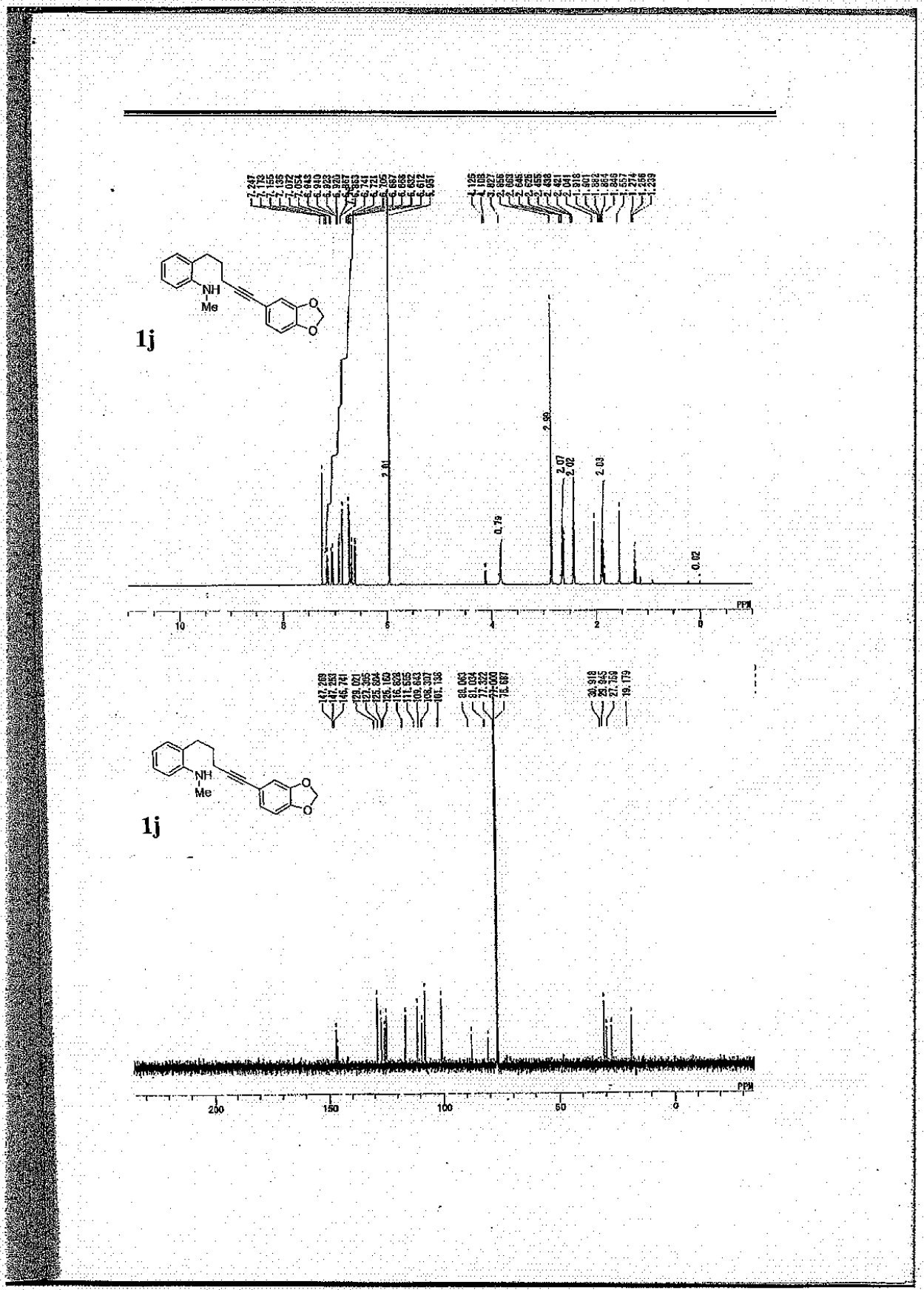




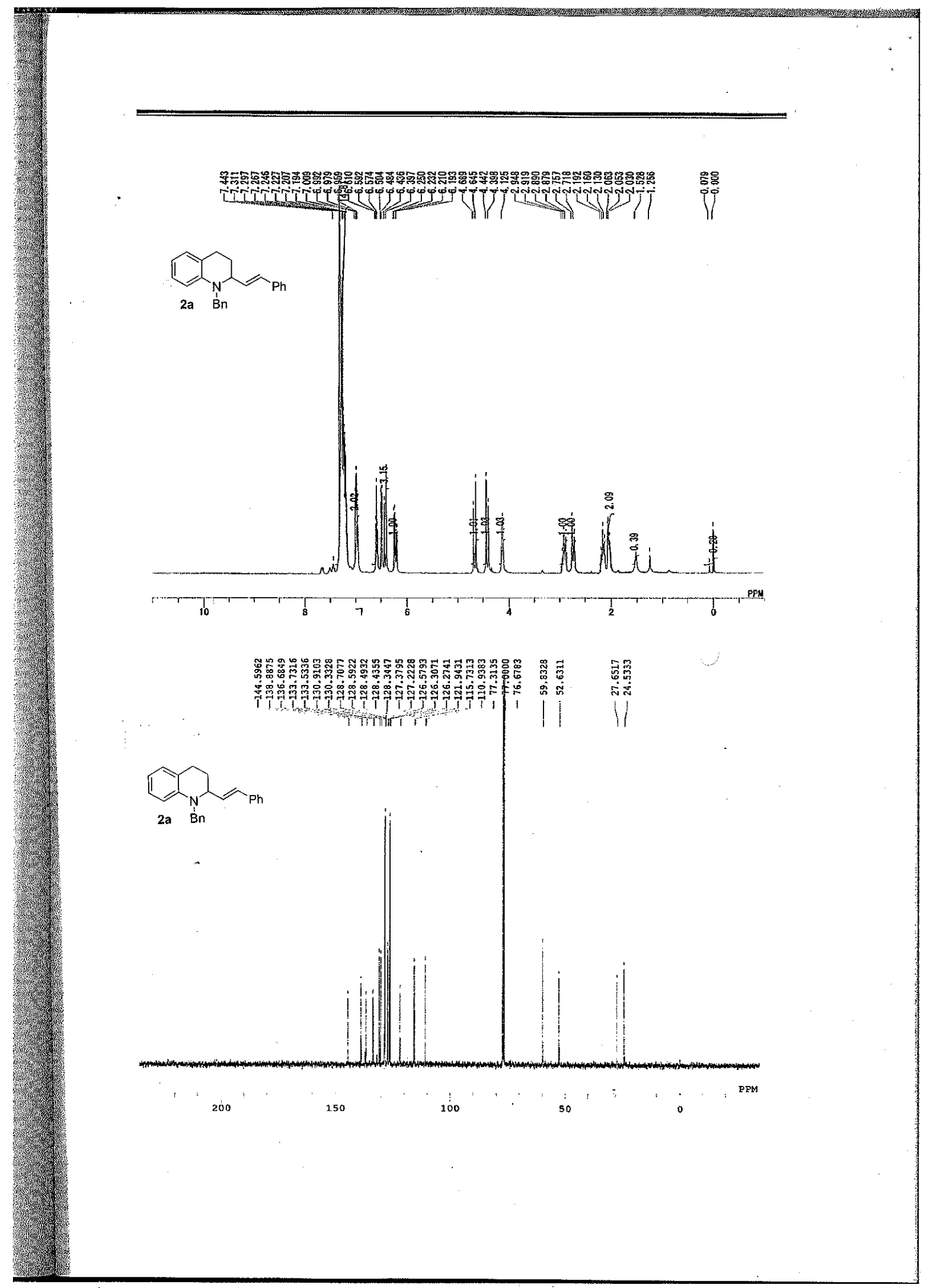




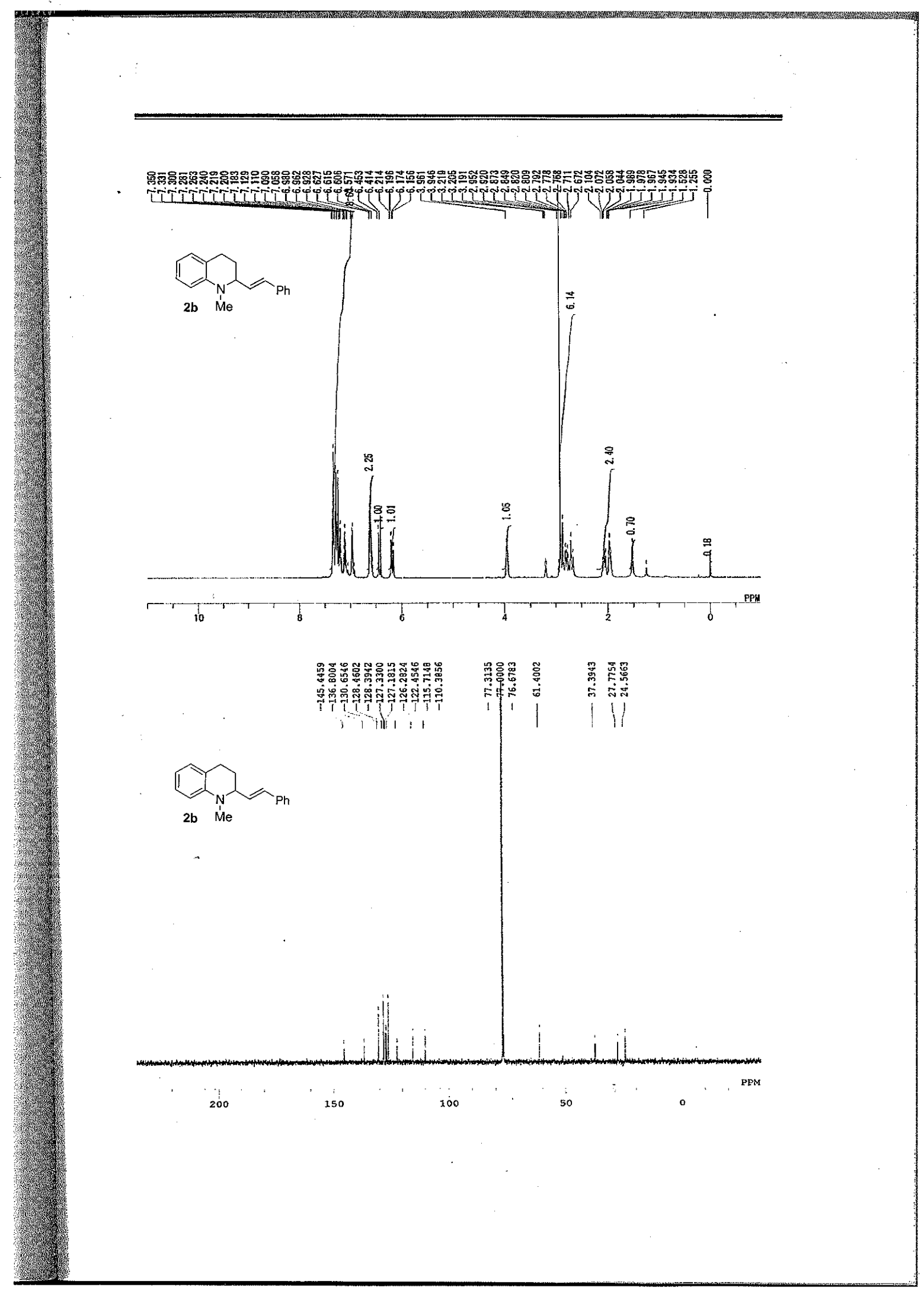




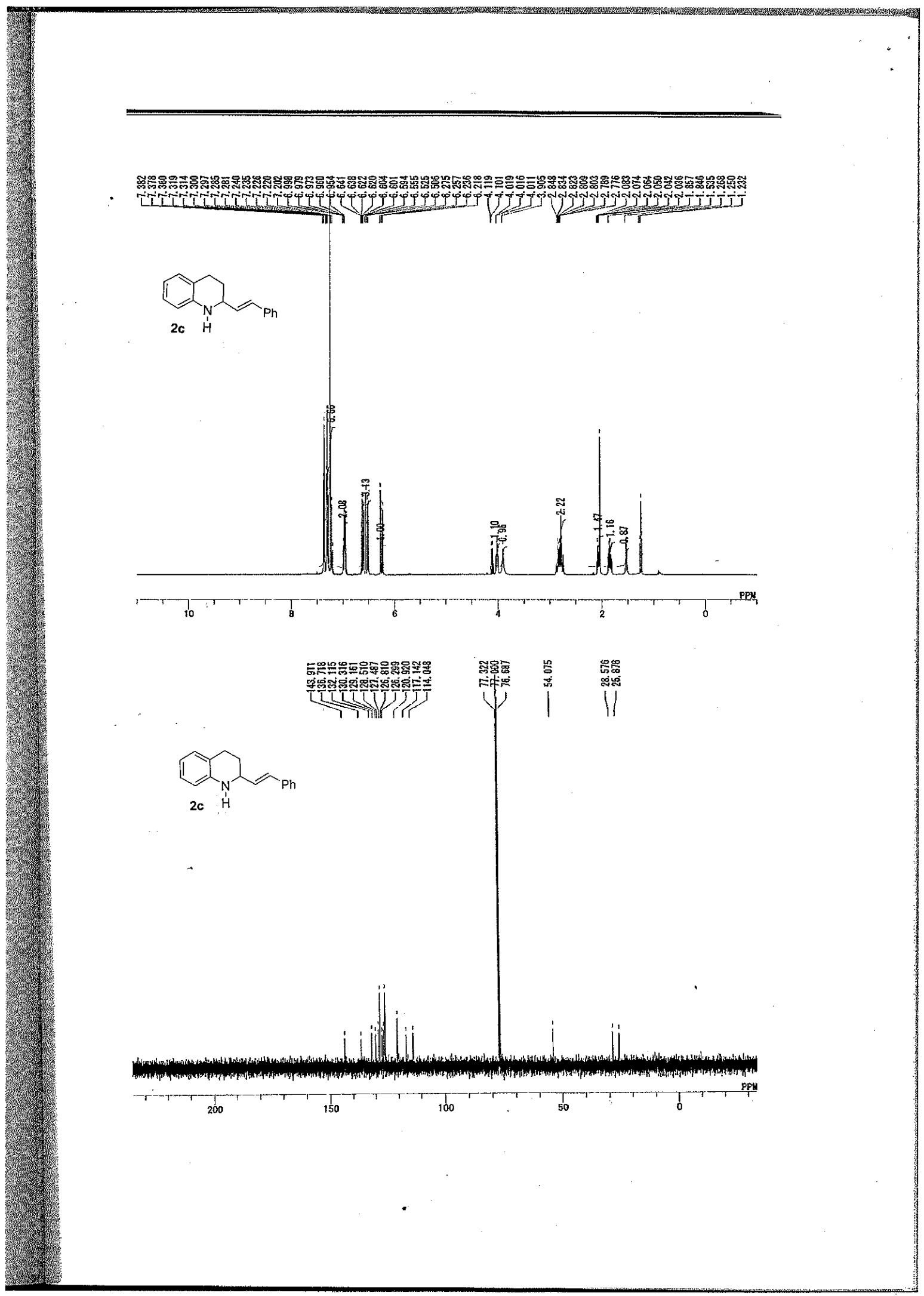




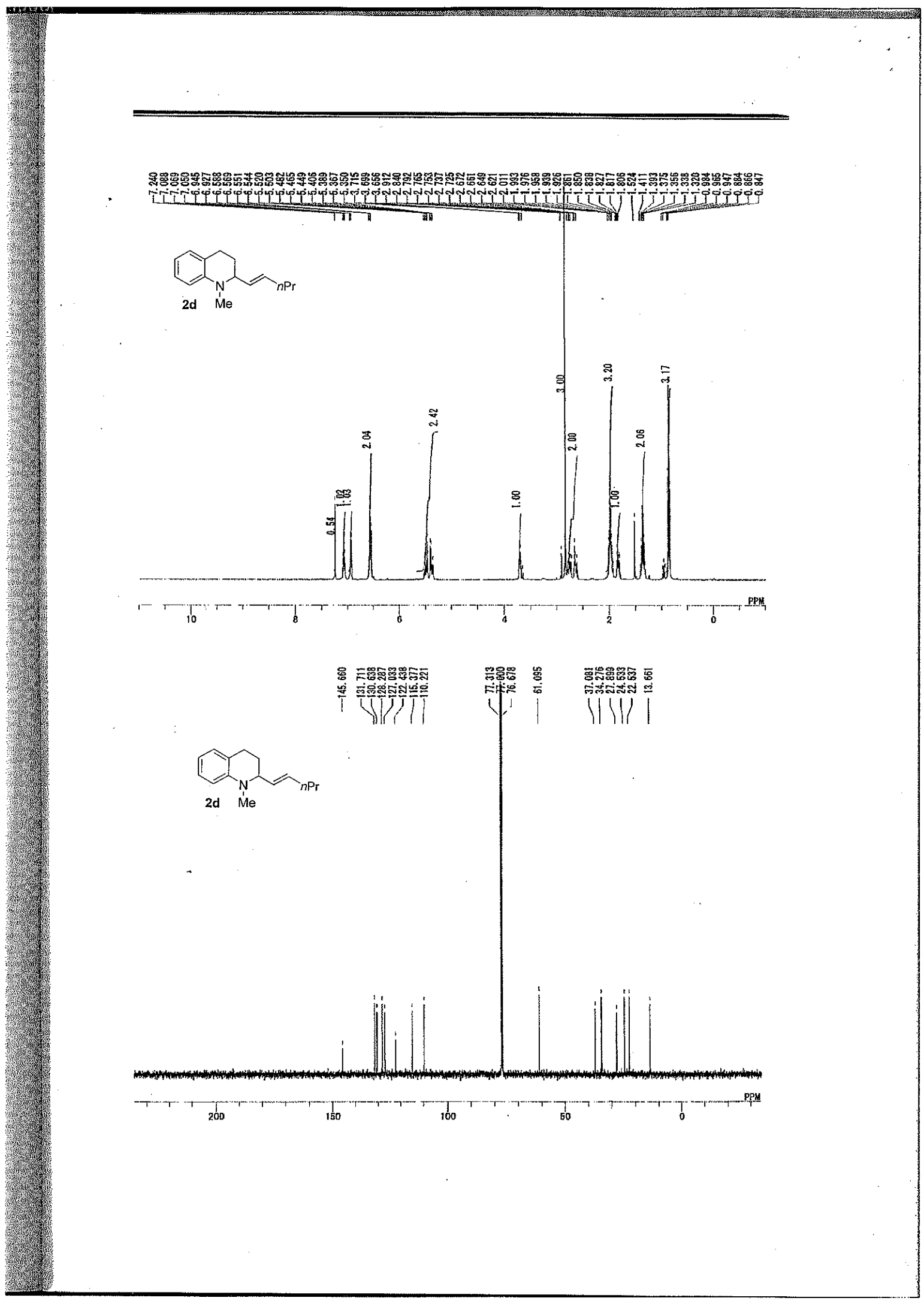




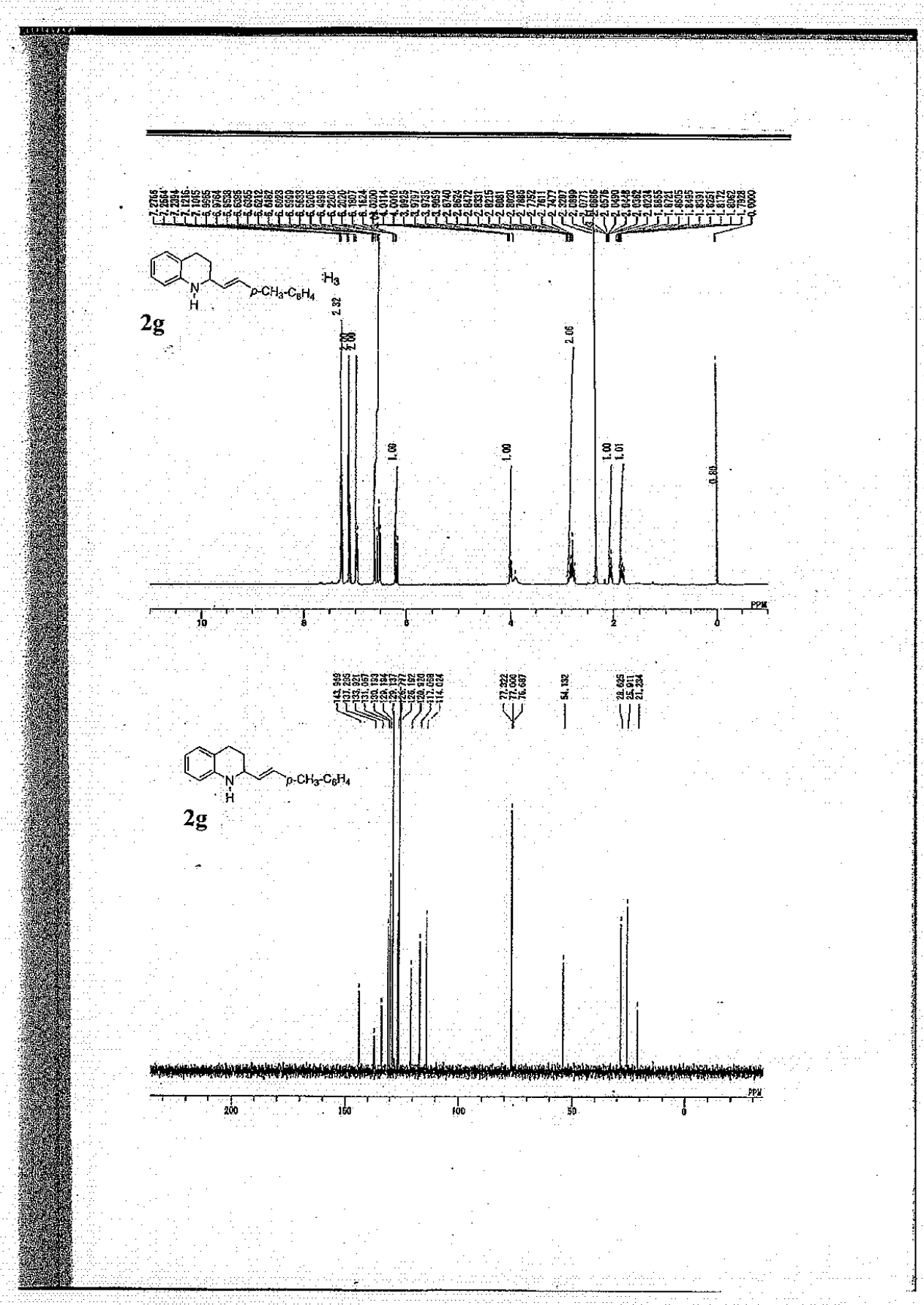




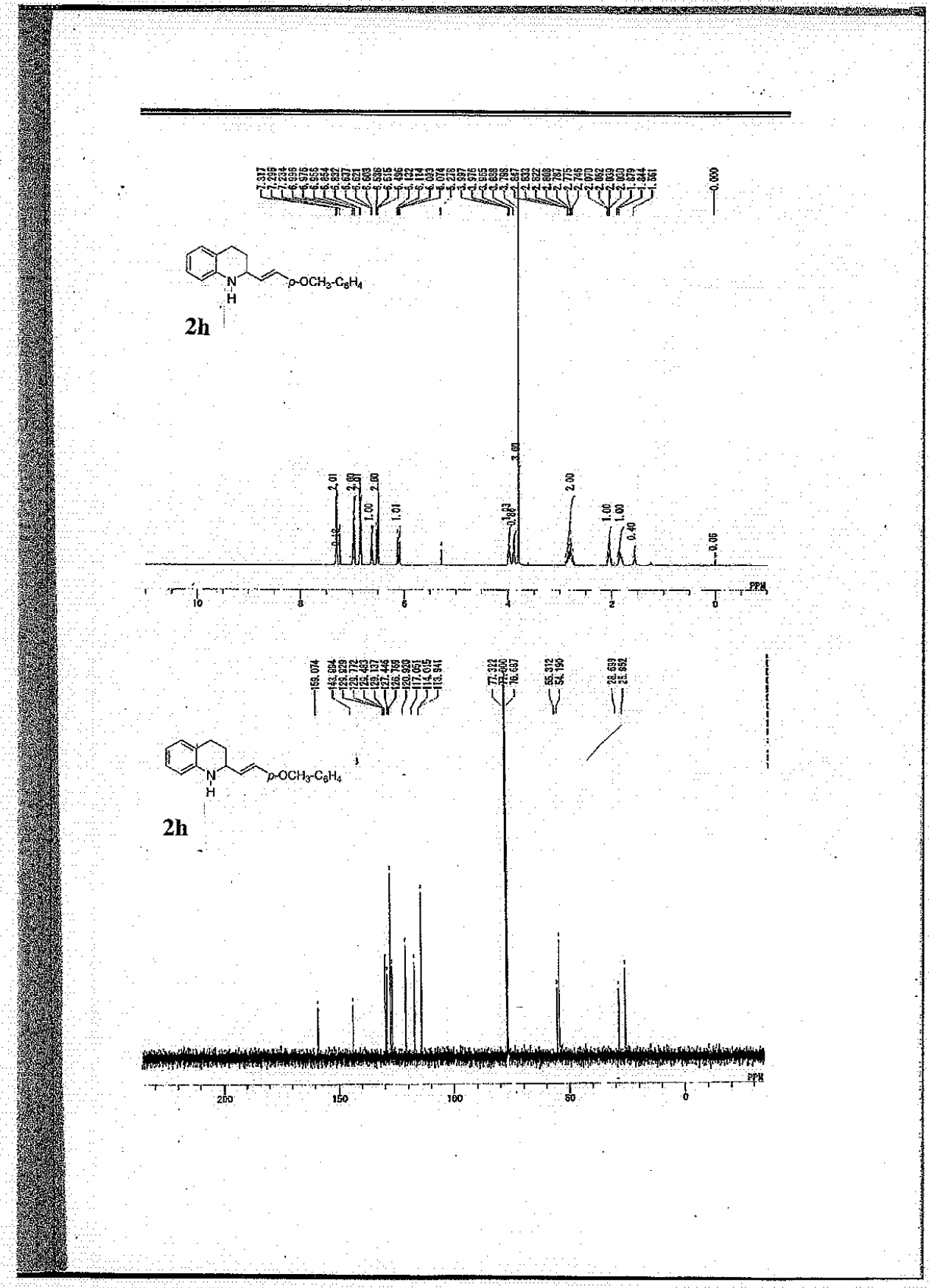




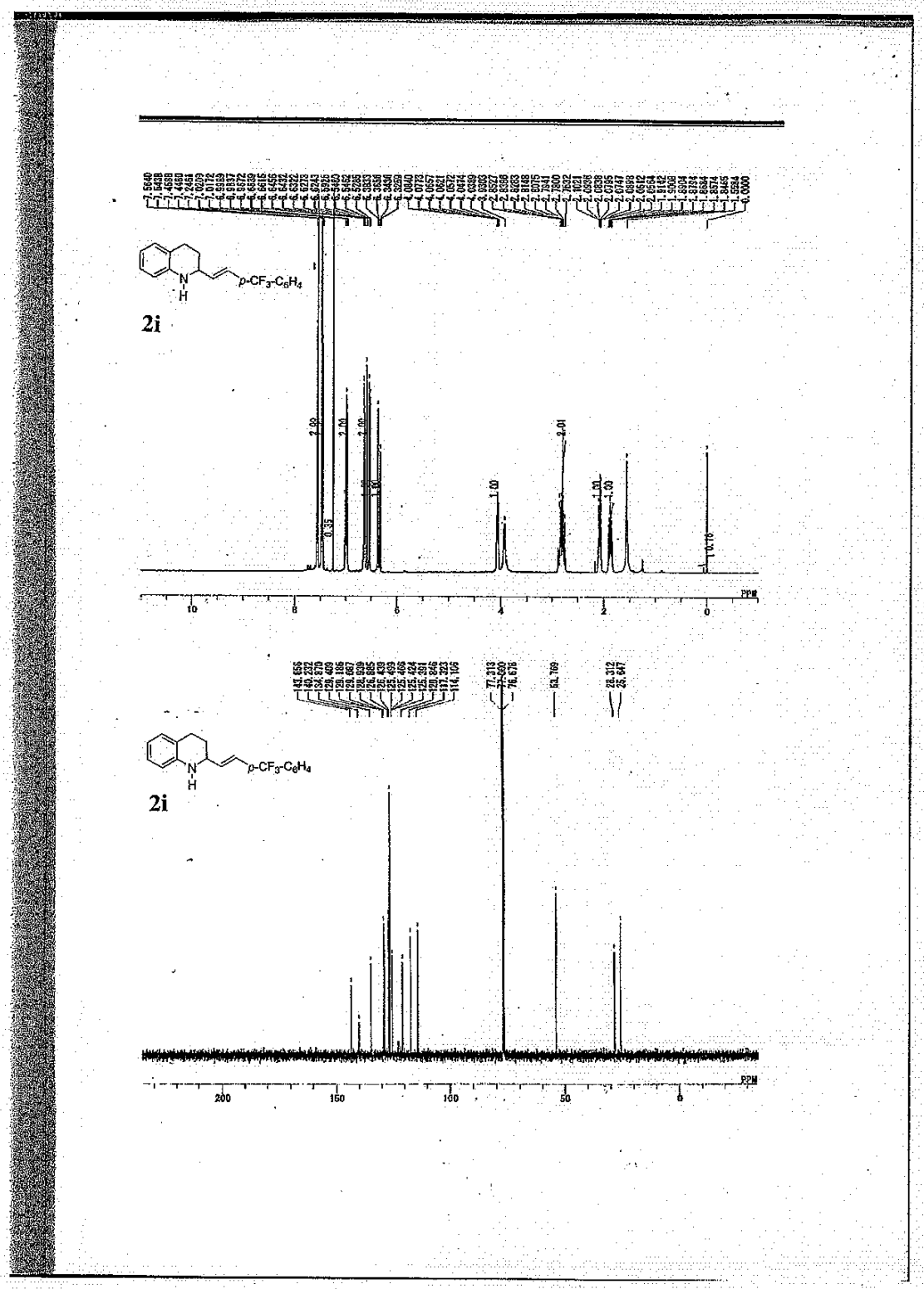




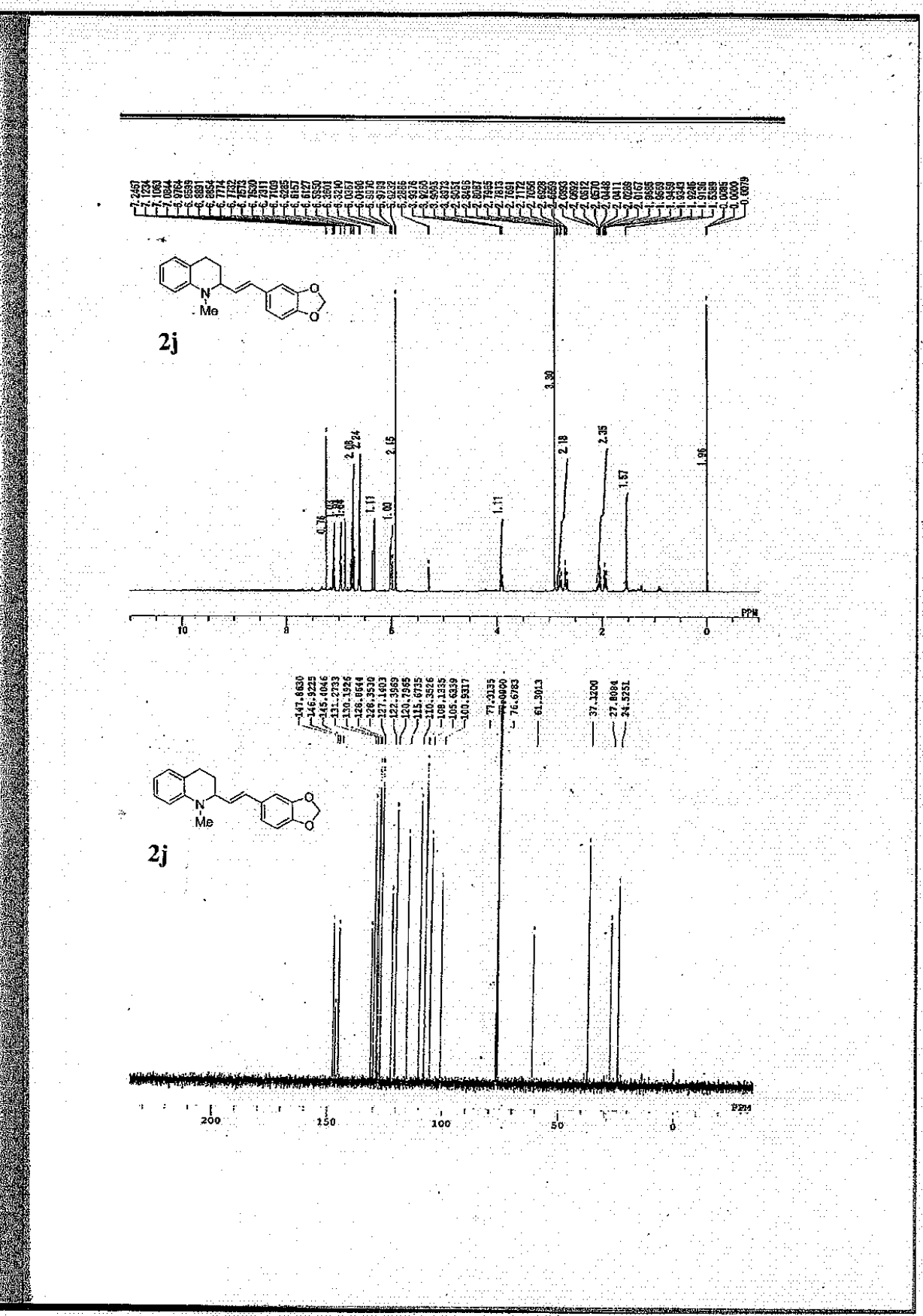




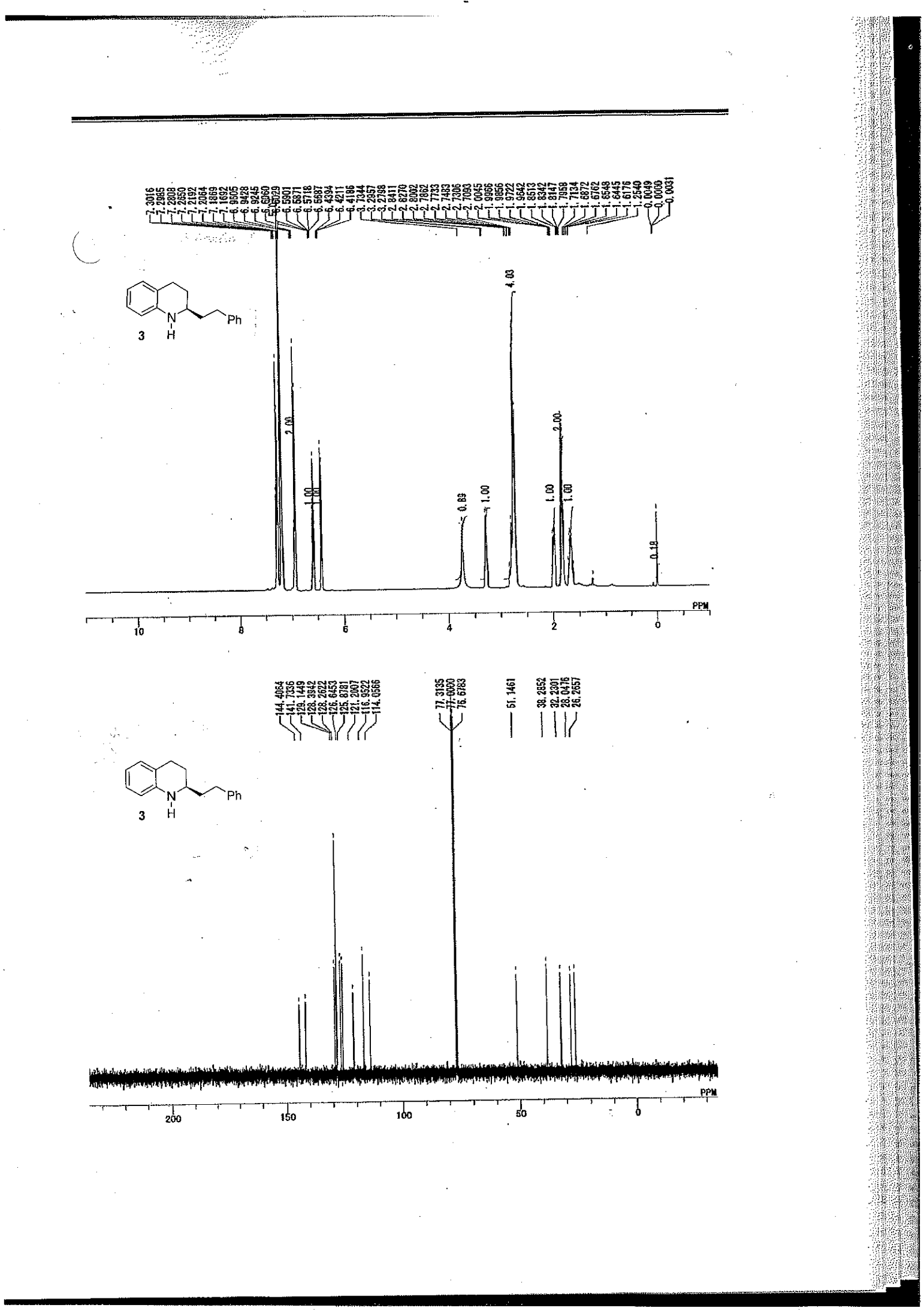




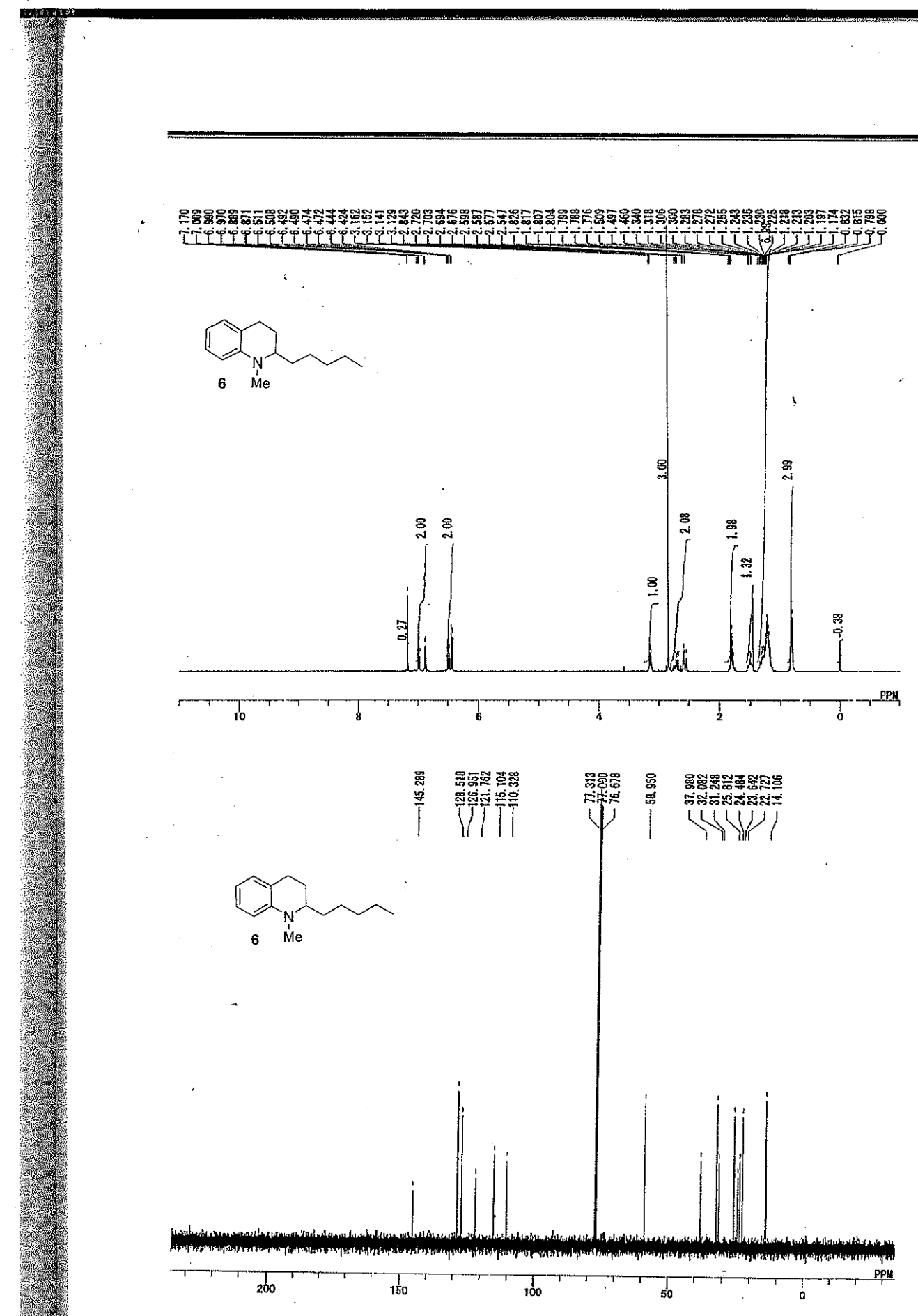




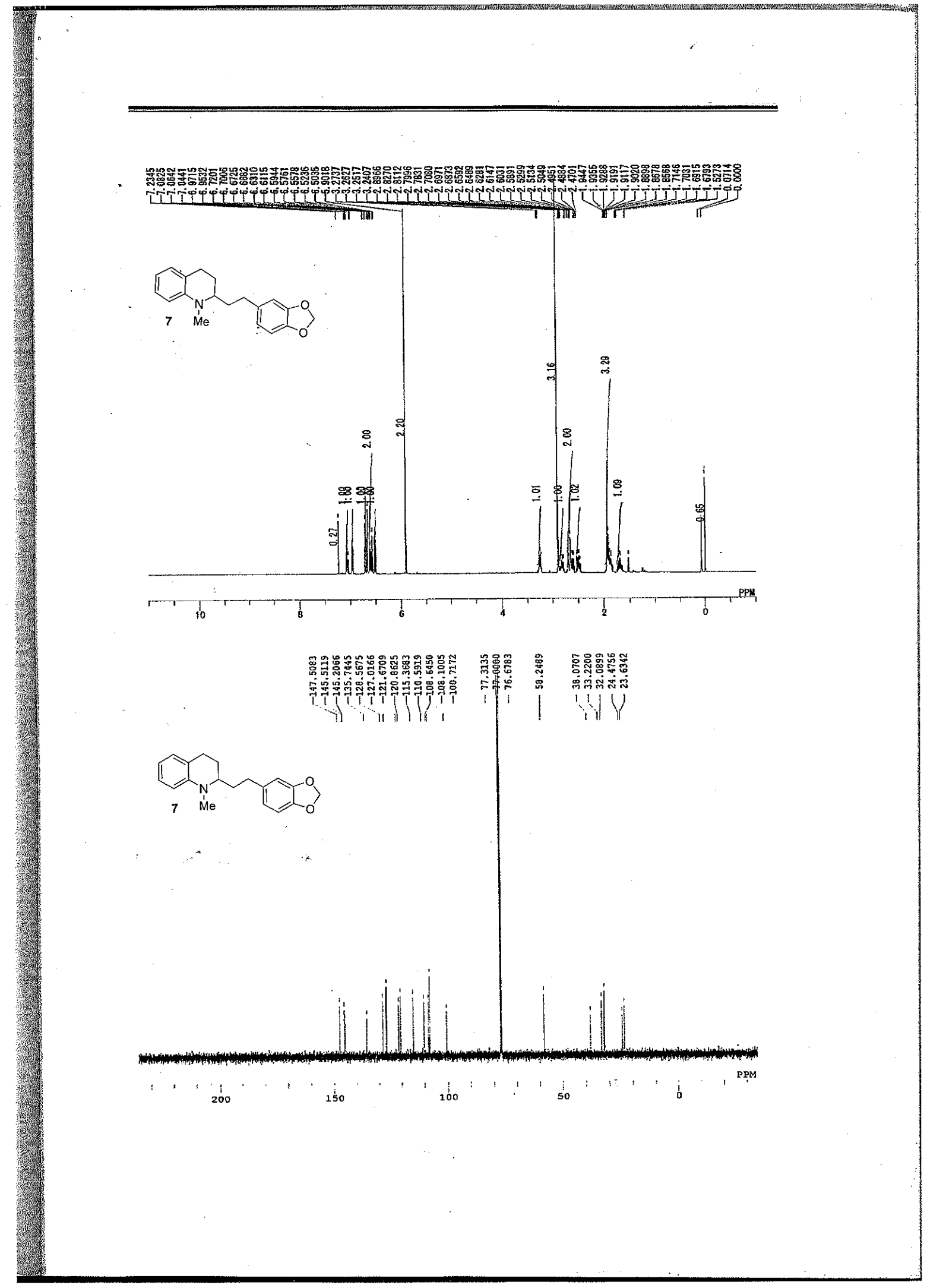

